Preprint typeset in JHEP style - HYPER VERSION

\title{
SCALE-INVARIANT ALTERNATIVES TO GENERAL RELATIVITY
}

\author{
Diego Blas*, Mikhail Shaposhnikov ${ }^{\dagger}$ and Daniel Zenhäusern ${ }^{\ddagger}$ \\ Institut de Théorie des Phénomènes Physiques, \\ École Polytechnique Fédérale de Lausanne, \\ CH-1015, Lausanne, Switzerland
}

\begin{abstract}
We study the general class of gravitational field theories constructed on the basis of scale invariance (and therefore absence of any mass parameters) and invariance under transverse diffeomorphisms (TDiff), which are the 4-volume conserving coordinate transformations. We show that these theories are equivalent to a specific type of scalar-tensor theories of gravity (invariant under all diffeomorphisms) with a number of properties, making them phenomenologically interesting. They contain, in addition to the dimensionless coupling constants of the original theory, an arbitrary dimensionful parameter $\Lambda_{0}$. This parameter is associated with an integration constant of the equations of motion, similar to the arbitrary cosmological constant appearing in unimodular gravity. We focus on the theories where Newton's constant and the electroweak scale emerge from the spontaneous breaking of scale invariance and are unrelated to $\Lambda_{0}$. The massless particle spectrum of these theories contains the graviton and a new particle - dilaton. For $\Lambda_{0}=0$, the massless dilaton has only derivative couplings to matter fields and the bounds on the existence of a 5 th force are easily satisfied. As for the matter fields, we determine the conditions leading to a renormalizable low-energy theory. If $\Lambda_{0} \neq 0$, scale invariance is broken. The arbitrary constant $\Lambda_{0}$ produces a "run-away" potential for the dilaton. As a consequence, the dilaton can act as a dynamical dark energy component. We elucidate the origin of the cosmological constant in the class of theories under consideration and formulate the condition leading to its absence. If this condition is satisfied, dark energy is purely dynamical and associated to the dilaton.
\end{abstract}

Keywords: Scalar Tensor Theories, Unimodular Gravity, Scale Invariance, Dilaton, Dark Energy.

\footnotetext{
*diego.blas@epfl.ch

${ }^{\dagger}$ mikhail.shaposhnikov@epfl.ch

${ }^{\ddagger}$ daniel.zenhaeusern@epfl.ch
} 


\section{Contents}

1. Introduction 1

2. TDiff invariant theories of gravity 5

2.1 Equivalent Diff invariant theories 6

2.2 Classical backgrounds and local degrees of freedom 8

3. Scale-invariant TDiff theories 10

3.1 Classical backgrounds and local degrees of freedom 14

3.2 Interactions and separation of scales 16

3.3 Dependence on the choice of variables and exact renormalizability 18

4. Including gauge bosons 20

4.1 Local degrees of freedom 22

4.2 Interactions and separation of scales 23

5. Coupling to fermionic matter 25

6. Application to the Standard Model 26

7. Particular choices of the theory-defining functions 27

7.1 Polynomial TDF 28

7.2 TDF leading to Abelian Higgs model plus a decoupled dilaton 30

7.3 TDF reproducing scale-invariant unimodular gravity 31

8. The case $\Lambda_{0} \neq 0$, cosmology and dilaton interactions 33

9. Conclusions 35

\section{Introduction}

General Relativity (GR) and the Standard Model of particle physics (SM) are characterized by two very different energy scales: the Planck mass $M_{P}$ (related to Newton's constant as $\left.M_{P}=\left(8 \pi G_{N}\right)^{-1 / 2}=2.4 \cdot 10^{18} \mathrm{GeV}\right)$ in the case of $\mathrm{GR}$ and the vacuum expectation value of the Higgs field in the $\mathrm{SM}, v \simeq 250 \mathrm{GeV} .^{1}$ In theories where a scalar field $\phi$ interacts "non-minimally" with the scalar curvature $R$ through a term $\xi \phi^{2} R$, the Planck mass can be generated dynamically [1, 2. In such theories, the Planck scale and the electroweak scale

\footnotetext{
${ }^{1}$ One can add to these scales a cosmological constant $\Lambda \sim 10^{-47} \mathrm{GeV}^{4}$ whose attribution to one or the other sector (GR or SM) is not understood.
} 
may have a common origin. A minimal option to realize this idea is to identify the scalar field $\phi$ with the Higgs field of the SM and choose the constant $\xi \sim M_{P} / M_{W} \sim 10^{16}$. In this case, the origin of the Planck scale is related to the electroweak symmetry breaking and the existence of a very large dimensionless constant [3, 4]. However, in this theory the Higgs field almost completely decouples from the other fields of the SM [3, 4, leading to contradiction with the precision tests of the electroweak theory. Moreover, the validity of theories with such large dimensionless parameters remains unclear. Therefore, adding extra fields to the SM and GR seems unavoidable for the realization of the "one-scale" scenario. The addition of new fields is further motivated by the fact that it allows to implement the idea of a "no-scale" scenario (see below).

In [5] two of us (M. S. and D. Z.) proposed an extension of the SM and GR containing an extra real scalar field $\chi$ - dilaton - and containing no absolute energy scale. Earlier works with similar ideas, but different in a number of essential points, include [6, 7, 8]. The Lagrangian of the model was fixed with the following principles:

i) The action does not contain terms with more than two derivatives.

ii) The action is invariant under global scale transformations

$$
\Phi(x) \mapsto \lambda^{d_{\Phi}} \Phi(\lambda x)
$$

where $\Phi$ stands for the different fields in the action (scalar, spinor, vector and gravitational), $\lambda$ is an arbitrary real constant and $d_{\Phi}$ is the canonical mass dimension of the field $\Phi$. The dilatational invariance is not preserved by the standard regularization schemes (such as dimensional regularization, Pauli-Villars regularization, cut-off regularization or lattice regularization) used in the Jordan frame formulation of the theory ${ }^{2}$. In fact, all these schemes introduce an explicit parameter with dimension of mass and hence break scale invariance. This eventually translates into the presence of anomalies. However, for the cases where scale invariance is spontaneously-broken, one can formulate modified regularization schemes that do not introduce any intrinsic mass parameter ${ }^{3}$, i.e. that are anomaly-free. The scale-invariant version of dimensional regularization is discussed in [11, 12], the field-dependent cut-off in [8] and lattice regularization in 13. The resulting effective, rather than fundamental, field theories [14] are scale-invariant to all orders of perturbation theory. The existence of scale-invariant regularization schemes suggests that exact scale invariance can still be a legitimate guiding principle for the construction of new theories.

iii) The particle physics part of the theory given in the Jordan frame is polynomial in the different fields.

\footnotetext{
${ }^{2}$ In the present context of scale-invariant theories, we define the Jordan frame as the frame in which the action is invariant under scale transformations of the form (1.1), and where the metric has zero mass dimension.

${ }^{3}$ Interestingly, these schemes become "standard" in the Einstein frame formulation of the theory [9, 10]. See also the comments after Eq. (3.18).
} 
iv) The Higgs-dilaton potential in the Jordan frame contains a flat direction and leads to spontaneous breaking of scale invariance. Any vacuum state on this flat direction gives rise to identical physics ${ }^{4}$. The Planck scale, particle masses, and quantum dimensional transmutation parameters like $\Lambda_{Q C D}$ are generated dynamically.

v) The space-time metric obeys the constraint $g=-1$, where $g=\operatorname{det} g_{\mu \nu}$, corresponding to Unimodular Gravity (UG) [16, 17, 18, 19, 20, 21, 22, 23], rather than conventional GR.

Besides realizing the "no-scale" scenario, this proposal solves (in a technical sense) the problem of stability of the Higgs mass against radiative corrections, which are kept small due to the exact scale invariance. For the precise meaning of this statement, see [12]. The requirement iv) leads to the absence of a cosmological constant term. The spontaneous breakdown of scale invariance naturally provides a mechanism for inflation. In addition, the almost massless and very weakly coupled dilaton acts as dynamical dark energy.

In the present work, we will consider the proposal of [5], and extend it to a setup in which the additional scalar degree of freedom appears as a part of the metric field. To introduce the setup, let us first recall that the spectrum of GR consist of just massless spin-two degrees of freedom (which is intimately related to the invariance of the theory under diffeomorphisms, called Diff invariance henceforth) [24]. The only other possible metric theory of gravity sharing this feature is unimodular gravity (UG) [16, 25]. Both possibilities are nearly equivalent (at least at the classical level): any solution of the UG equations of motion corresponds to a solution of the GR equations with a particular value of the cosmological constant $\Lambda=\Lambda_{0}$. The only difference is that the parameter $\Lambda$ in GR is a fundamental constant while $\Lambda_{0}$ in UG is an integration constant determined by initial conditions. One can go beyond GR by considering the (perhaps) more physical requirement of having a consistent metric theory including spin-two polarization. It can be proven 16, 25] that the minimal group of gauge invariance required to construct such a theory is the subgroup of coordinate transformations

$$
x^{\mu} \mapsto \tilde{x}^{\mu}(x), \text { with } J \equiv\left|\frac{\partial \tilde{x}^{\mu}}{\partial x^{\nu}}\right|=1,
$$

which is generated by the subalgebra of transverse vectors,

$$
\delta x^{\mu}=\xi^{\mu}, \quad \partial_{\mu} \xi^{\mu}=0 .
$$

We will refer to these transformations as transverse diffeomorphisms (TDiff) ${ }^{5}$. Under TDiff transformations the determinant of the metric $g$ transforms as a scalar. Even more, in

\footnotetext{
${ }^{4}$ It is important to distinguish this way of spontaneous breaking of scale invariance from the approach presented in [15]. The authors of 15] argued that the mere existence of cosmological evolution may be enough to provide an energy scale from which every other mass can be derived. It is, though, unclear whether this proposal can be made phenomenologically acceptable.

${ }^{5}$ There are different names for these transformations in the literature, including volume or area preserving diffeomorphisms. In this work we will use the name TDiff as it does not make any reference to the dimensionality of space-time. Besides, we will use the term "TDiff theories" for theories invariant under TDiff.
} 
TDiff theories $g$ generally corresponds to a propagating scalar degree of freedom, the only exceptions being GR and UG [25]. As has been argued in the past, a TDiff theory is not equivalent to standard scalar-tensor gravity but rather to UG plus a new scalar field, which is potentially massive 119,25$]$. The objective of this work is to study general properties of scale-invariant TDiff theories, and to show that they are phenomenologically viable. We mainly work with the classical theory.

The paper is organized as follows. We start in Section 2 with a discussion of TDiff gravity theories and provide a simple proof of their classical equivalence to Diff invariant theories of gravity with an arbitrary integration constant $\Lambda_{0}$. After discussing the background solutions, we explicitly show that a scalar degree of freedom is present in the gravitational sector. From this analysis, we clarify the role of $\Lambda_{0}$ as an extra initial condition representing a new coupling constant of a peculiar potential for the scalar field.

Next, the attention is turned to scale-invariant TDiff theories (Section 3). It is shown that TDiff invariance allows to choose potentials that lead to spontaneous breaking of scale invariance and thereby generate all energy scales of the theory. For the particle physics phenomenology we focus on the potentials that allow for a stable static background ${ }^{6}$, which in particular requires $\Lambda_{0}=0$.We find that the particle spectrum around such a background necessarily contains a massless scalar excitation, the Goldstone boson of the spontaneouslybroken scale symmetry. Since this particle can not play the role of the SM Higgs field, we include an additional scalar field (in a realistic theory this can be the complex scalar doublet of the SM.) After passing to the Einstein frame, we identify the massless field (dilaton) and the potentially massive field (Higgs boson). ${ }^{7}$ In the Einstein frame the original scale invariance, existing in the Jordan frame, is replaced by a shift symmetry of the dilaton field. As long as $\Lambda_{0}=0$, the shift symmetry is unbroken and the dilaton couples only derivatively. Hence, it easily avoids experimental bounds on the existence of a long-ranged 5th force. The case $\Lambda_{0} \neq 0$ is relevant for cosmological considerations (which are deferred to Section 8). Besides, for $\Lambda_{0} \neq 0$ the shift symmetry is broken by the presence of a new interaction term between the dilaton and the Higgs field with an interaction scale related to the integration constant.

In Sections 4 and a we include gauge fields and fermions in our considerations and define the conditions yielding a model with massive gauge vectors (potentials related to the Higgs model). These results are used in Section 6 to outline how the new framework can be applied to the Standard Model. In Section 7 we summarize the requirements to be imposed on the scale-invariant TDiff Lagrangians which lead to an acceptable low-energy phenomenology. In addition, we present particular examples of scale-invariant TDiff theories that satisfy these requirements. One of the examples corresponds to the model of [5].

Section 8 briefly discusses the case $\Lambda_{0} \neq 0$ and cosmological applications. We will show that for a certain class of potentials, the cosmological solutions are very similar to those found in the particular case discussed in [5]. Namely, the spontaneous breakdown of scale invariance due to the flat direction in the scalar potential dynamically generates

\footnotetext{
${ }^{6}$ Some theoretical arguments in its favor were given in 肳, 12 and we have nothing to add at the moment.

${ }^{7}$ The Einstein frame is defined as the frame in which the gravitational part of the action is given by the standard Einstein-Hilbert action.
} 
Newton's gravitational constant and particle masses and thereby provides a mechanism for inflation, whereas the breakdown of scale invariance due to the $\Lambda_{0}$-term leads to dynamical dark energy. We show here that in spite of the fact that $\Lambda_{0} \neq 0$, the dilaton practically decouples and thus evades all the constraints on extra forces.

We present our conclusions in Section 9 .

\section{TDiff invariant theories of gravity}

The group of invariance of Einstein's theory of General Relativity is the group of all diffeomorphisms (coordinate changes)

$$
x^{\mu} \mapsto \tilde{x}^{\mu}(x),
$$

whose infinitesimal form is

$$
x^{\mu} \mapsto x^{\mu}+\xi^{\mu}(x) .
$$

We will refer to the group of all diffeomorphisms with the abbreviation Diff. If gravity is described by a symmetric metric $g_{\mu \nu}$, Diff invariance, together with the requirement that the field equations should contain no higher than second derivatives, uniquely fixes the form of the gravitational action. Diff invariance also dictates how matter fields are coupled to gravity (with the possibility of non-minimal couplings), resulting in an extremely successful theory [26].

Looking for theoretical alternatives to GR, one can consider the question of finding the minimal group of gauge invariance giving rise to a satisfactory theory of gravitation (in particular including spin- 2 excitations $)^{8}$. The answer to this question is the TDiff group that was introduced in (1.2), cf. 116, 25). This is one of the motivations for exploring TDiff gravity, see e.g. [19, 25, 29, 30].

Unlike Diff invariance, TDiff invariance does not uniquely fix the form of the gravitational action. In particular, the action can contain arbitrary functions of the metric determinant, $g$, since it is a scalar under TDiff. The most general TDiff invariant action for gravity containing no higher than second derivatives is therefore ${ }^{9}$

$$
\mathcal{S}_{T D}=\int \mathrm{d} x^{4} \sqrt{-g}\left(-\frac{1}{2} M^{2} f(-g) R-\frac{1}{2} M^{2} \mathcal{G}(-g) g^{\mu \nu} \partial_{\mu} g \partial_{\nu} g-M^{4} v(-g)\right),
$$

where $f(-g), \mathcal{G}(-g)$ and $v(-g)$ are arbitrary functions and $M$ is an a priori arbitrary mass scale. For the previous action to be defined (in particular for the existence of $g^{\mu \nu}$ ), it is necessary that $-g>0$, which we will assume henceforth. The couplings between gravity and matter based on TDiff invariance are also much less restricted than in the case of Diff invariance. Just like the gravitational part of the action, they can contain arbitrary functions

\footnotetext{
${ }^{8}$ Here we will only consider the case of Lorentz invariant theories. For viable theories of gravity without Lorentz invariance see e.g. 27, 28].

${ }^{9}$ We will follow Weinberg's conventions: $\eta_{\mu \nu}=\operatorname{diag}(-1,1,1,1), R_{\beta \gamma \delta}^{\alpha}=\partial_{\delta} \Gamma_{\beta \gamma}^{\alpha}+\Gamma_{\beta \gamma}^{\lambda} \Gamma_{\lambda \delta}^{\alpha}-(\gamma \leftrightarrow \delta)$. Finally $R_{\mu \nu}=R_{\mu \alpha \nu}^{\alpha}$. In this conventions, if $\tilde{g}_{\mu \nu}=\Omega^{2} g_{\mu \nu}$,

$$
\tilde{R}=\Omega^{-2}\left(R+\frac{3}{\sqrt{-g}} \partial_{\mu}\left(\sqrt{-g} g^{\mu \nu} \partial_{\nu} \ln \Omega^{2}\right)+\frac{3}{2} g^{\mu \nu} \partial_{\mu} \ln \Omega^{2} \partial_{\nu} \ln \Omega^{2}\right) .
$$
}


of $g$. We will refer to the arbitrary functions of $g$ as "Theory-Defining Functions" (TDF). Ultimately, all TDF will be restricted by theoretical and phenomenological considerations.

The action $S_{T D}$ describes in general three propagating degrees of freedom, the graviton plus a new scalar. There are two particular choices for the arbitrary functions that enhance the TDiff invariance by an additional local invariance such that the scalar degree of freedom is absent $\mid 25 \|$. The first one obviously corresponds to GR $(f=$ const., $v=$ const., $\mathcal{G}=0)$. The second one corresponds to choosing the functions such that the action is invariant under local (Weyl) rescalings of the metric $g_{\mu \nu} \mapsto e^{2 \sigma(x)} g_{\mu \nu}$, where $\sigma(x)$ is an arbitrary function $\left(f=(-g)^{-1 / 4}, v=0, \mathcal{G}=-\frac{3}{32}(-g)^{-9 / 4}\right)$. In this second case (sometimes called WTDiff), the action depends on the metric only through the unimodular metric $\hat{g}_{\mu \nu}=g_{\mu \nu}(-g)^{-1 / 4}$. Therefore, this case exactly corresponds to UG.

Except for the previous cases, we expect the theory to have arbitrary $\mathcal{G}$ and $v$, which implies the existence of a new scalar degree of freedom in the field $g_{\mu \nu}$. Depending on its mass this will have different phenomenological consequences (in particular for searches of 5 th forces). As we will explicitly show in the next section, the theory can be reformulated in the more standard framework where the additional degree of freedom appears as a new type of "matter" (or source for the standard GR metric) that can mediate interactions between other fields of the SM. Thus, the distinction between gravity and matter becomes ambiguous in these theories. In particular, this allows us to relate the Higgs field of the SM to the determinant of the metric, and to interpret the "new" interactions within the SM framework.

\subsection{Equivalent Diff invariant theories}

It proves very convenient to reformulate TDiff invariant theories as Diff invariant theories, where the extra degree of freedom appears explicitly. In this section we will make use of the Stückelberg formalism to achieve this goal (see also [19, 23, 31, 32 for related works).

Let us consider the generic TDiff invariant Lagrangian (2.3). To start with, note that one can always add an arbitrary constant $\Lambda_{0}$ to this Lagrangian, without changing the theory. Next, one can transform the associated action to an arbitrary coordinate frame by performing a generic Diff transformation. The resulting action is

$$
\begin{aligned}
\mathcal{S}_{e}=\int \mathrm{d}^{4} x \sqrt{-g}( & -\frac{1}{2} M^{2} f(-g / a) R-\frac{1}{2} M^{2} \mathcal{G}(-g / a) g^{\mu \nu} \partial_{\mu}(-g / a) \partial_{\nu}(-g / a) \\
& \left.-M^{4} v(-g / a)-\frac{\Lambda_{0}}{\sqrt{-g / a}}\right)
\end{aligned}
$$

where $a(x) \equiv J(x)^{-2}, J(x)$ being the Jacobian of the Diff transformation, and $\Lambda_{0}$ is the aforementioned arbitrary constant. The action (2.4) is classically equivalent to (2.3) and the equations of motion for $g_{\mu \nu}$ are identical. Let us now promote $a(x)$ to a dynamical field (commonly called Stückelberg, Goldstone or Compensator field) and let it transform under the Diff (2.2) like the determinant of the metric, i.e. as

$$
\delta_{\xi} a=\xi^{\mu} \partial_{\mu} a+2 a \partial_{\mu} \xi^{\mu}
$$


As a consequence, the action (2.4) is invariant under Diff,

$$
\int \mathrm{d}^{4} y\left(\frac{\delta \mathcal{S}_{e}}{\delta a(y)} \delta_{\xi} a(y)+\frac{\delta \mathcal{S}_{e}}{\delta g_{\mu \nu}(y)} \delta_{\xi} g_{\mu \nu}(y)\right)=0
$$

where

$$
\delta_{\xi} g_{\mu \nu}=\nabla_{\mu} \xi_{\nu}+\nabla_{\nu} \xi_{\mu}
$$

If the metric satisfies its equations of motion, the previous identity is reduced to

$$
\int \mathrm{d}^{4} y \sqrt{a} \xi^{\mu} \partial_{\mu}\left(\sqrt{a} \frac{\delta \mathcal{S}_{e}}{\delta a}\right)=0 .
$$

This identity is valid for arbitrary $\xi^{\mu}$ and hence the equations of motion of the metric imply

$$
\frac{\delta \mathcal{S}_{e}}{\delta a}=\frac{C_{0}}{\sqrt{a}},
$$

where $C_{0}$ is an arbitrary integration constant. The left-hand side of these equations contains a term proportional to $\Lambda_{0}$, which has exactly the same form as the term on the right-hand side. Hence, the term of the right-hand side can always be absorbed by a redefinition of the arbitrary constant $\Lambda_{0}$, resulting in

$$
\frac{\delta \mathcal{S}_{e}}{\delta a}=0
$$

This is enough to prove that the equations of motion derived from (2.4), considering $g_{\mu \nu}$ and $a$ as independent fields, are equivalent to those derived from (2.3), where only $g_{\mu \nu}$ is varied. By construction, the new action has an additional local invariance. In the gauge $a=1$ (which we assume to be achievable) the solutions of the new equations are exactly the same as those gotten from (2.3). Solutions derived in a gauge $a \neq 1$ also correspond to the solutions of (2.3), however now written in different coordinates.

We will refer to the model characterized by the Lagrangian density $\mathcal{L}_{e}$ in (2.4) as the equivalent Diff invariant theory. Also in the rest of this paper the subscript $e$ will be used in this sense. Let us now define the field

$$
\sigma \equiv-g / a>0
$$

which is a scalar under all diffeomorphisms, and rewrite the Lagrangian as

$$
\mathcal{L}_{e}=\sqrt{-g}\left(-\frac{1}{2} M^{2} f(\sigma) R-\frac{1}{2} M^{2} \mathcal{G}(\sigma) g^{\mu \nu} \partial_{\mu} \sigma \partial_{\nu} \sigma-M^{4} v_{\Lambda_{0}}(\sigma)\right)
$$

where

$$
v_{\Lambda_{0}}(\sigma)=M^{4} v(\sigma)+\frac{\Lambda_{0}}{\sqrt{\sigma}} .
$$

The theory formulated this way reduces to (2.3) after imposing the gauge condition $-g=\sigma$ (corresponding to $a=1$ ). For any other gauge conditions with $-g \neq \sigma$ (which may be more convenient for other reasons), it still corresponds to the original TDiff theory but written in new coordinates related to the original ones by a transformation with Jacobian $J \neq 1$. 
The appearance of a new parameter $\Lambda_{0}$ is a general feature of TDiff theories (see also |33 for other theories of gravity involving arbitrary integration constants). For $f(-g)=$ $(-g)^{-1 / 4} *$ cst. (like in pure UG [16, 17, 18, 23n) it plays the role of a cosmological constant. In all other cases, $\Lambda_{0}$ leads to a new specific potential term for the scalar field $\sigma$. At this point, we would like to stress that $\Lambda_{0}$ is a parameter characterizing the solution of the equations of motion and is not a fundamental coupling constant in the action (2.3). At the classical level, $\Lambda_{0}$ should be understood as an additional global degree of freedom which turns out to be a constant of the motion: once the initial conditions and $\Lambda_{0}$ are chosen, the evolution proceeds identically in both, scalar-tensor theories of gravity and TDiff gravity ${ }^{10}$.

Quantum mechanically, the relation between both theories is more subtle (see e.g. [21, 23, 34, 35). Being a global degree of freedom, $\Lambda_{0}$ can be treated in two different ways in the quantum theory. First, one can consider the projected case, where $\Lambda_{0}$ is fixed to a certain value. This case is identical to GR [34]. One could also consider $\Lambda_{0}$ as an integration variable in the path integral formulation of the theory. However, in the absence of a welldefined path integral formulation of the theory, the results of this approach, though very interesting, should be considered as preliminary (see, e.g., [35, 36]).

\subsection{Classical backgrounds and local degrees of freedom}

In this section, we consider the maximally symmetric background solutions of the theory described by (2.12) and determine the conditions, under which they are perturbatively stable. By a maximally symmetric background solution we mean a solution of the classical equations of motion, which corresponds to constant fields in the particle physics sector of the theory and a maximally symmetric geometry, i.e. Minkowski (flat), de Sitter (dS) or Anti de Sitter (AdS) space-time. The existence of such a ground state may be essential for a consistent quantization of the theory. In order to find such solutions it is convenient to first rewrite the theory in the Einstein frame (E-frame), where the scalar field $\sigma$ is minimally coupled to the metric. We define the E-frame metric as

$$
\begin{aligned}
& \tilde{g}_{\mu \nu}=\Omega^{2} g_{\mu \nu}, \\
& \tilde{g}^{\mu \nu}=\Omega^{-2} g^{\mu \nu}, \\
& \Omega^{2}=f(\sigma),
\end{aligned}
$$

in terms of which the Lagrangian (2.12) reads

$$
\mathcal{L}_{e}=\sqrt{-\tilde{g}}\left(-\frac{1}{2} M^{2} \tilde{R}-\frac{1}{2} \mathcal{K}(\sigma) M^{2} \tilde{g}^{\mu \nu} \partial_{\mu} \sigma \partial_{\nu} \sigma-V_{\Lambda_{0}}(\sigma)\right)
$$

where

$$
\mathcal{K}(\sigma)=\frac{\mathcal{G}(\sigma)}{f(\sigma)}+\frac{3}{2}\left(\frac{f^{\prime}(\sigma)}{f(\sigma)}\right)^{2}, \quad V_{\Lambda_{0}}(\sigma)=\frac{v_{\Lambda_{0}}(\sigma)}{f(\sigma)^{2}} .
$$

\footnotetext{
${ }^{10}$ In this sense, the equations of motion of the single Lagrangian (2.3) correspond to those of a whole family of Lagrangians 2.12 with different values of $\Lambda_{0}$.
} 
For the previous transformation to make sense as a field redefinition between two field theories defined perturbatively around a certain background $\sigma_{0}$, one should assume

$$
f\left(\sigma_{0}\right) \neq 0
$$

This is at the same time the condition leading to an induced gravitational scale and weakly interacting spin-two excitations around this background (cf. ((2.12)). We will assume it henceforth.

For a constant scalar field $\sigma=\sigma_{0}$, the equations of motion imply

$$
\begin{aligned}
& \Lambda_{0}=2 M^{4} \sigma_{0}^{3 / 2}\left(\frac{v^{\prime}\left(\sigma_{0}\right) f\left(\sigma_{0}\right)-2 f^{\prime}\left(\sigma_{0}\right) v\left(\sigma_{0}\right)}{f\left(\sigma_{0}\right)+4 \sigma_{0} f^{\prime}\left(\sigma_{0}\right)}\right), \\
& \tilde{R}=-4 M^{2}\left(\frac{v\left(\sigma_{0}\right)+2 \sigma_{0} v^{\prime}\left(\sigma_{0}\right)}{f\left(\sigma_{0}\right)\left[f\left(\sigma_{0}\right)+4 \sigma_{0} f^{\prime}\left(\sigma_{0}\right)\right]}\right) .
\end{aligned}
$$

Unless the right-hand side vanishes, the first equation can be understood as an equation for $\sigma_{0}$ in terms of $\Lambda_{0}$. The second equation shows that the solution may be flat, dS or AdS, depending on the TDF (and on $\Lambda_{0}$ through $\left.\sigma_{0}\right)$. In the degenerate case $f\left(\sigma_{0}\right)+4 \sigma_{0} f^{\prime}\left(\sigma_{0}\right)=0$ (which, in particular, corresponds to UG), a maximally symmetric background solution only exists, if there is a value $\sigma_{0}$, for which $v\left(\sigma_{0}\right)+2 \sigma_{0} v^{\prime}\left(\sigma_{0}\right)=0$. The classical ground state is then given by $\sigma=\sigma_{0}$ and $\tilde{R}=-4 V_{\Lambda_{0}} M^{-2}$. Again, depending on the TDF, respectively the value of $\Lambda_{0}$, the corresponding maximally symmetric space-time is flat, dS or AdS.

Thus, in a TDiff theory containing the metric as the only field, maximally symmetric background solutions always exist, independently of the TDF (except may be in the degenerate case). Flat space-time is a solution, provided

$$
v\left(\sigma_{0}\right)+2 \sigma_{0} v^{\prime}\left(\sigma_{0}\right)=0
$$

For the study of the propagating degrees of freedom we will focus on the case in which flat space-time is a solution,

$$
\tilde{g}_{\mu \nu}=\eta_{\mu \nu}, \quad \sigma=\sigma_{0}, \quad \Lambda_{0}=-M^{4} v\left(\sigma_{0}\right) \sqrt{\sigma_{0}}
$$

and introduce the perturbations

$$
\tilde{g}_{\mu \nu}=\eta_{\mu \nu}+\frac{\tilde{h}_{\mu \nu}}{M}, \quad \sigma=\sigma_{0}+\frac{\varsigma}{M} .
$$

The part of the Lagrangian (2.14) quadratic in perturbations reads ${ }^{11}$

$$
\mathcal{L}_{e}^{Q}=\frac{1}{2} \tilde{\mathcal{L}}_{E H}^{Q}-\frac{1}{2} \mathcal{K}^{(0)} \eta^{\mu \nu} \partial_{\mu} \varsigma \partial_{\nu} \varsigma-\frac{1}{2} V_{\Lambda_{0}}^{(2)} M^{-2} \varsigma^{2},
$$

where the first term in (2.21) is the standard quadratic Einstein-Hilbert Lagrangian

$$
\tilde{\mathcal{L}}_{E H}^{Q}=-\frac{1}{4} \partial_{\rho} \tilde{h}_{\mu \nu} \partial^{\rho} \tilde{h}^{\mu \nu}+\frac{1}{2} \partial^{\nu} \tilde{h}_{\mu \nu} \partial^{\rho} \tilde{h}^{\mu}{ }_{\rho}-\frac{1}{2} \partial_{\mu} \tilde{h} \partial_{\nu} \tilde{h}^{\mu \nu}+\frac{1}{4} \partial_{\mu} \tilde{h} \partial^{\mu} \tilde{h}
$$

\footnotetext{
${ }^{11}$ Here and in the rest of the paper, we use the notation $F^{(n)}=\left.\frac{d^{n} F(\sigma)}{d \sigma^{n}}\right|_{\sigma=\sigma 0}$ for the derivatives of functions evaluated at the background field value .
} 
with indices raised and lowered with the Minkowski metric $\eta_{\mu \nu}$ and $\tilde{h} \equiv \tilde{h}_{\mu}^{\mu}$. This term describes two massless tensor degrees of freedom. From (2.21) one can see that whenever $\mathcal{K}^{(0)}=\mathcal{K}\left(\sigma_{0}\right) \neq 0$, the theory also contains a scalar degree of freedom ${ }^{12}$. In that case, the scalar part can be brought to canonical form by defining the canonically normalized field

$$
\varsigma_{c}=\sqrt{\left|\mathcal{K}^{(0)}\right|} \varsigma
$$

We get

$$
\mathcal{L}_{e}^{Q}=\frac{1}{2} \tilde{\mathcal{L}}_{E H}^{Q}-\epsilon_{\varsigma} \frac{1}{2} \partial_{\mu} \varsigma_{c} \partial^{\mu} \varsigma_{c}-\frac{m_{\varsigma}^{2}}{2} \varsigma_{c}^{2}
$$

where

$$
\epsilon_{\varsigma} \equiv \operatorname{sign}\left(\mathcal{K}^{(0)}\right), \quad m_{\varsigma}^{2} \equiv \epsilon_{\varsigma} \frac{V_{\Lambda_{0}}^{(2)}}{\mathcal{K}^{(0)}} M^{-2}
$$

The perturbations around the background (2.19) will be well-behaved provided that:

- The scalar field $\varsigma_{c}$ has a positive definite kinetic term (absence of ghosts): $\mathcal{K}^{(0)}>0$.

- The field $\varsigma_{c}$ has positive or zero mass (absence of tachyons): $V_{\Lambda_{0}}^{(2)} \geq 0$.

Finally, on top of the terms quadratic in the perturbations there is obviously a series of interaction terms. We will get interested in those terms in the upcoming sections, where we consider the phenomenology of different types of fields coupled to TDiff gravity.

\section{Scale-invariant TDiff theories}

In this section we will focus on scale-invariant TDiff theories including scalar fields only. Other SM fields will be introduced in the subsequent sections.

Assuming that the metric has a non-zero scaling dimension $d_{g} \neq 0$, the Lagrangian (2.3) is invariant under the scale transformation

$$
g_{\mu \nu}(x) \mapsto \lambda^{d_{g}} g_{\mu \nu}(\lambda x),
$$

provided that the TDF satisfy,

$$
f(-g)=f_{0}(-g)^{\frac{2-d_{g}}{4 d_{g}}}, \quad \mathcal{G}(-g)=\mathcal{G}_{0}(-g)^{-2+\frac{2-d_{g}}{4 d_{g}}}, \quad v(-g)=v_{0}(-g)^{\frac{2-d_{g}}{2 d_{g}}},
$$

where $f_{0}, \mathcal{G}_{0}$ and $v_{0}$ are arbitrary constants.

The scaling dimension of the metric can be changed by performing the TDiff compatible field redefinition ( $\alpha$ is a real constant)

$$
g_{\mu \nu} \mapsto(-g)^{\alpha} g_{\mu \nu}
$$

Thus, different scaling dimensions correspond to equivalent theories. In particular, we could set $d_{g}=2$. The scale transformation with $d_{g}=2$ corresponds to a diffeomorphism

\footnotetext{
${ }^{12}$ Both for GR $(f(\sigma)=1$ and $\mathcal{G}(\sigma)=v(\sigma)=0)$ and for UG $\left(f=\sigma^{-1 / 4}, \mathcal{G}=-\frac{3}{32} \sigma^{-9 / 4}, v=0\right)$ one finds $\mathcal{K}^{(0)}=0$ and hence these theories only contain the two massless tensor degrees of freedom.
} 
and hence any Diff invariant theory is scale-invariant in the aforementioned sense (notice that the opposite is not true: not any scale-invariant TDiff theory is Diff invariant). In particular, GR corresponds to the Lagrangians with TDF (3.2) satisfying $\frac{\mathcal{G}_{0}}{f_{0}}=-\frac{3}{2}\left(\frac{2-d_{g}}{4 d_{g}}\right)^{2}$ and $v_{0}=0$. Let us mention that $\mathrm{UG}$ is not invariant under (3.1).

Following the discussion in Section 2.2, we may look for maximally symmetric solutions for the TDF (3.2). Recalling that $\sigma>0$, one finds that $f\left(\sigma_{0}\right)+4 \sigma_{0} f^{\prime}\left(\sigma_{0}\right) \neq 0$ for all values of $\sigma_{0}$. Hence, maximally symmetric background solutions always exist. These solutions spontaneously break the scale symmetry. The condition (2.18) for the existence of the flat space-time solution is fulfilled only if $v_{0}=0$. Except for the case corresponding to GR, the spectrum around the flat background solution contains a propagating massless scalar degree of freedom. It represents the Goldstone boson of the spontaneously broken scale invariance.

The above theory is interesting because of its uniqueness. However, it is not enough for our purposes, since we want to build a theory containing a massive scalar field that plays the role of the SM Higgs field. In view of this, we will now consider the possibility of adding an extra real scalar field, $\phi$. A scale-invariant TDiff theory including $g_{\mu \nu}$ and $\phi$ will be invariant under the transformations

$$
g_{\mu \nu}(x) \mapsto \lambda^{d_{g}} g_{\mu \nu}(\lambda x), \quad \phi(x) \mapsto \lambda^{d_{\phi}} \phi(\lambda x)
$$

By a field redefinition of the type

$$
g_{\mu \nu} \mapsto(-g)^{\alpha} \phi^{\beta} g_{\mu \nu}, \quad \phi \mapsto(-g)^{\gamma} \phi^{\delta}
$$

compatible with the TDiff invariance, the scaling dimensions of the fields can always be changed to different values. In other words, the way one attributes scaling dimensions to the different fields merely corresponds to the choice of field variables. Without loss of generality, we will choose the scaling dimensions to correspond to the usual canonical mass dimensions, i.e. $d_{g}=0$ and $d_{\phi}=1$, for which the scale transformation is

$$
g_{\mu \nu}(x) \mapsto g_{\mu \nu}(\lambda x), \quad \phi(x) \mapsto \lambda \phi(\lambda x)
$$

An alternative choice of the scaling dimensions would be $d_{g}=2$ and $d_{\phi}=0$. In this case, the scaling dimensions of the fields correspond to their tensorial rank. This choice reveals an interesting property of scale-invariant TDiff theories: the group of invariance including TDiff and the scale transformations where all fields have scaling dimension equal to their tensorial rank can be identified as a subgroup of Diff (see also the comments after Eq. (3.3)). In other words, the symmetry group consisting of TDiff plus global scale transformations constitutes a subgroup of the full Diff group.

Supplementing the model (2.3) by a real scalar field and imposing invariance under the 
transformation (3.6), one finds the action ${ }^{13}$

$$
\begin{aligned}
\mathcal{S}=\int \mathrm{d} x^{4} \sqrt{-g}\left(-\frac{1}{2} \phi^{2} f(-g) R-\right. & \frac{1}{2} \phi^{2} \mathcal{G}_{g g}(-g)(\partial g)^{2}-\frac{1}{2} \mathcal{G}_{\phi \phi}(-g)(\partial \phi)^{2} \\
& \left.+\mathcal{G}_{g \phi}(-g) \phi \partial g \cdot \partial \phi-\phi^{4} v(-g)\right) .
\end{aligned}
$$

Here, and in many of the upcoming expressions, in order to shorten notations, we do no longer write Lorentz indices explicitly. The implicit contractions of Lorentz indices are done with the metric $g_{\mu \nu}$ if the Lagrangian is in the J-frame and with $\tilde{g}_{\mu \nu}$ if it is in the Eframe. Like in the theory containing only the metric field (Section 2), the Lagrangian (3.7) contains arbitrary functions (TDF) of the metric determinant $g$. The dependence on the scalar field $\phi$ is dictated by scale invariance. Note, however, that the situation is different if one chooses variables such that $d_{g}=2$ and $d_{\phi}=0$. In that case, scale invariance dictates the dependence of the Lagrangian on $g$, while the arbitrary functions solely depend on $\phi$.

Using the Stückelberg formalism illustrated in Section 2.1 we can write down the Lagrangian of the equivalent Diff invariant theory of (3.7) as

$$
\begin{aligned}
\frac{\mathcal{L}_{e}}{\sqrt{-g}}=-\frac{1}{2} \phi^{2} f(\sigma) R- & \frac{1}{2} \phi^{2} \mathcal{G}_{g g}(\sigma)(\partial \sigma)^{2}-\frac{1}{2} \mathcal{G}_{\phi \phi}(\sigma)(\partial \phi)^{2} \\
& -\mathcal{G}_{g \phi}(\sigma) \phi \partial \sigma \cdot \partial \phi-\phi^{4} v(\sigma)-\frac{\Lambda_{0}}{\sqrt{\sigma}} .
\end{aligned}
$$

For $\Lambda_{0}=0$, the corresponding action is invariant under $(\overline{3.6})$ supplemented by the transformation of $\sigma$, i.e.

$$
g_{\mu \nu}(x) \mapsto g_{\mu \nu}(\lambda x), \quad \phi(x) \mapsto \lambda \phi(\lambda x), \quad \sigma(x) \mapsto \sigma(\lambda x)
$$

In fact, in this case, (3.8) is also invariant under the internal transformation

$$
g_{\mu \nu}(x) \mapsto \lambda^{2} g_{\mu \nu}(x), \quad \phi(x) \mapsto \lambda^{-1} \phi(x) .
$$

A non-zero $\Lambda_{0}$ breaks these symmetries. Thus, scale-invariant TDiff theories naturally produce a unique symmetry-breaking potential term. This should be contrasted with the situation in generic Diff invariant theories, where such a term could only be introduced ad hoc. In other words, starting from a Diff invariant theory, there would be no reason to include in (3.8) the term proportional to $\Lambda_{0}$ without also including all other possible terms breaking the scale symmetry (3.9).

The Lagrangian (3.8) can be transformed to the Einstein frame (provided $\phi^{2} f(\sigma) \neq 0$ ), with the help of a conformal transformation

$$
\tilde{g}_{\mu \nu}=\Omega^{2} g_{\mu \nu}, \quad \tilde{g}^{\mu \nu}=\Omega^{-2} g^{\mu \nu}, \quad \Omega^{2}=\frac{\phi^{2} f(\sigma)}{M^{2}} .
$$

\footnotetext{
${ }^{13}$ Terms with arbitrarily many derivatives can be included in a scale-invariant way, if one allows for $\phi$ to appear in the denominator. We will assume that, if present, these terms are suppressed by a large energy scale.
} 
It takes the form

$$
\begin{aligned}
\frac{\mathcal{L}_{e}}{\sqrt{-\tilde{g}}}=-\frac{1}{2} M^{2} \tilde{R} & -\frac{1}{2} M^{2} \mathcal{K}_{\sigma \sigma}(\sigma)(\partial \sigma)^{2}-\frac{1}{2} M^{2} \mathcal{K}_{\phi \phi}(\sigma)(\partial \ln (\phi / M))^{2} \\
& -M^{2} \mathcal{K}_{\sigma \phi}(\sigma) \partial \sigma \cdot \partial \ln (\phi / M)-M^{4} V(\sigma)-\frac{M^{4} \Lambda_{0}}{\phi^{4} f(\sigma)^{2} \sqrt{\sigma}},
\end{aligned}
$$

where

$$
\begin{array}{ll}
\mathcal{K}_{\sigma \sigma}(\sigma)=\frac{\mathcal{G}_{g g}(\sigma)}{f(\sigma)}+\frac{3}{2}\left(\frac{f^{\prime}(\sigma)}{f(\sigma)}\right)^{2}, & \mathcal{K}_{\phi \phi}(\sigma)=\frac{\mathcal{G}_{\phi \phi}(\sigma)}{f(\sigma)}+6, \\
\mathcal{K}_{\sigma \phi}(\sigma)=\frac{\mathcal{G}_{g \phi}(\sigma)}{f(\sigma)}+3 \frac{f^{\prime}(\sigma)}{f(\sigma)} \quad, \quad V(\sigma) & =\frac{v(\sigma)}{f(\sigma)^{2}} .
\end{array}
$$

Except for the term proportional to $\Lambda_{0}$, the E-frame action is invariant under scale transformations with $d_{\tilde{g}}=2$ and $d_{\phi}=1 .{ }^{14}$ That is why, in the scale-invariant part, $\phi$ can only enter in the combination

$$
\partial_{\mu} \ln (\phi / M) .
$$

This can also be understood from the fact that in the E-frame the transformation (3.10) becomes

$$
\tilde{g}_{\mu \nu}(x) \mapsto \tilde{g}_{\mu \nu}(x), \quad \phi(x) \mapsto \lambda^{-1} \phi(x)
$$

The kinetic term for the scalar fields can be diagonalized by redefining the fields as ${ }^{15}$

$$
\tilde{\sigma}=\int_{\sigma_{0}}^{\sigma} \mathrm{d} \sigma^{\prime} \sqrt{\left|\frac{\mathcal{K}_{\sigma \sigma}\left(\sigma^{\prime}\right) \mathcal{K}_{\phi \phi}\left(\sigma^{\prime}\right)-\mathcal{K}_{\sigma \phi}\left(\sigma^{\prime}\right)^{2}}{\mathcal{K}_{\phi \phi}\left(\sigma^{\prime}\right)}\right|}, \quad \tilde{\phi}=M\left(\ln \frac{\phi}{M}+\int_{\sigma_{0}}^{\sigma} \mathrm{d} \sigma^{\prime} \frac{\mathcal{K}_{\sigma \phi}\left(\sigma^{\prime}\right)}{\mathcal{K}_{\phi \phi}\left(\sigma^{\prime}\right)}\right) .
$$

Note that we chose the integration constant such that $\tilde{\sigma}\left(\sigma_{0}\right)=0$ and kept $\sigma_{0}$ arbitrary for the moment. After this field redefinition (which is always solvable in perturbation theory) the Lagrangian simplifies to

$$
\frac{\mathcal{L}_{e}}{\sqrt{-\tilde{g}}}=-\frac{1}{2} M^{2} \tilde{R}-\frac{1}{2} \epsilon_{\sigma} M^{2}(\partial \tilde{\sigma})^{2}-\frac{1}{2} \tilde{\mathcal{K}}_{\phi \phi}(\tilde{\sigma})(\partial \tilde{\phi})^{2}-M^{4} \tilde{V}(\tilde{\sigma})-\Lambda_{0} \tilde{\mathcal{K}}_{\Lambda_{0}}(\tilde{\sigma}) \exp \left(-\frac{4 \tilde{\phi}}{M}\right)
$$

where $\epsilon_{\sigma}=\operatorname{sign}\left(\frac{\mathcal{K}_{\sigma \sigma}(\sigma) \mathcal{K}_{\phi \phi}(\sigma)-\mathcal{K}_{\sigma \phi}(\sigma)^{2}}{\mathcal{K}_{\phi \phi}(\sigma)}\right)$, and the different functions are obtained by expressing $\sigma$ as a function of $\tilde{\sigma}$,

$$
\tilde{V}(\tilde{\sigma})=V(\sigma), \quad \tilde{\mathcal{K}}_{\phi \phi}(\tilde{\sigma})=\mathcal{K}_{\phi \phi}(\sigma), \quad \tilde{\mathcal{K}}_{\Lambda_{0}}(\tilde{\sigma})=\frac{\exp \left(4 \int_{\sigma_{0}}^{\sigma} \mathrm{d} \sigma^{\prime} \frac{\mathcal{K}_{\sigma \phi}\left(\sigma^{\prime}\right)}{\mathcal{K}_{\phi \phi}\left(\sigma^{\prime}\right)}\right)}{f(\sigma)^{2} \sqrt{\sigma}} .
$$

After the field redefinition (3.16), the scale transformation for $\phi$ translates into the invariance under global shifts of the dilaton field, $\tilde{\phi} \mapsto \tilde{\phi}+\lambda$. This can be understood as the E-frame manifestation of scale invariance in the J-frame. If $\Lambda_{0}=0$, the dilaton is exactly

\footnotetext{
${ }^{14}$ Note that in the equivalent Diff invariant formulation, the action is exactly invariant under scale transformations with $d_{g}=d_{\tilde{g}}=2$ and $d_{\phi}=0$ as these transformations are a part of Diff.

${ }^{15}$ Here we assume that both $\mathcal{K}_{\phi \phi}(\sigma)$ and $\frac{\mathcal{K}_{\sigma \sigma}(\sigma) \mathcal{K}_{\phi \phi}(\sigma)-\mathcal{K}_{\sigma \phi}(\sigma)^{2}}{\mathcal{K}_{\phi \phi}(\sigma)}$ are non-zero.
} 
massless, and interacts with the field $\tilde{\sigma}$ (matter field) only through derivatives. In other words, it does not lead to measurable long-range interactions (for experimental bounds on light dilatons see e.g. [37, 38]).

This Lagrangian, when considered at the quantum field theory level, can be regularized by the standard procedures, such as dimensional or Pauli-Villars regularization. The subtraction procedure is then consistent with the shift symmetry and Diff invariance, i.e. this theory is anomaly free even if one uses the standard regularization schemes. Transforming the E-frame theory (action (3.17) plus counter-terms) back to the J-frame will result in a quantum theory with exact scale invariance. In dimensional regularization the exact invariance will be due to a field-dependent subtraction point, as described in [11, 12], while if Pauli-Villars or lattice regularizations are used, it will be due to a field-depend mass, respectively lattice spacing $[13$.

\subsection{Classical backgrounds and local degrees of freedom}

In this subsection we perform the analysis of maximally symmetric solutions and degrees of freedom for scale-invariant TDiff theories. As in Section 2.2, we will perform the analysis in the E-frame and assume that scale invariance is spontaneously broken; in particular

$$
\phi_{0}^{2} f\left(\sigma_{0}\right) \neq 0
$$

Once the previous condition is satisfied, Newton's constant (and other scales of the theory) are induced by the non-zero value of $\phi_{0}$.

For maximally symmetric solutions, the scalar fields must be constant. Contrary to the previous case (cf. Section 2.2), this automatically sets the constant $\Lambda_{0}=0$ (other possibilities relevant for cosmological applications will be considered in Section 8). After setting $\sigma=\sigma_{0}$, the equation of motion for $\sigma$ yields the condition

$$
V^{\prime}\left(\sigma_{0}\right)=0
$$

or, in terms of the original TDF,

$$
f\left(\sigma_{0}\right) v^{\prime}\left(\sigma_{0}\right)-2 f^{\prime}\left(\sigma_{0}\right) v\left(\sigma_{0}\right)=0
$$

If this condition holds, the remaining equations for the background fields are

$$
\phi=\phi_{0}, \quad \tilde{R}=-4 M^{2} \frac{v\left(\sigma_{0}\right)}{f\left(\sigma_{0}\right)^{2}}, \quad \Lambda_{0}=0
$$

where $\phi_{0}$ is not fixed by the equations of motion (as a consequence of scale invariance). For $v\left(\sigma_{0}\right) \neq 0$, the background will correspond to a dS or AdS space, while the Minkowski background is obtained for $v\left(\sigma_{0}\right)=0$. This, together with the constraint (3.21), implies that in the scale-invariant theory with spontaneous symmetry breaking, the existence of a Minkowski background requires (compare with (2.18))

$$
v\left(g_{0}\right)=v^{\prime}\left(g_{0}\right)=0 \text {. }
$$


Once these conditions are satisfied, the Lagrangian (3.17) has a background solution with

$$
\tilde{g}_{\mu \nu}=\eta_{\mu \nu}, \quad \tilde{\sigma}=0, \quad \tilde{\phi}=\tilde{\phi}_{0}, \quad \Lambda_{0}=0,
$$

where $\tilde{\phi}_{0}$ is an arbitrary real constant. We define the perturbations to the background as

$$
\begin{aligned}
& \tilde{g}_{\mu \nu}=\eta_{\mu \nu}+\frac{\tilde{h}_{\mu \nu}}{M}, \\
& \tilde{\sigma}=\frac{\varsigma}{M}, \\
& \tilde{\phi}=\tilde{\phi}_{0}+\frac{\varphi}{\sqrt{\mid \tilde{\mathcal{K}}_{\phi \phi}^{(0) \mid}}} .
\end{aligned}
$$

In the rest of Section 3, Lorentz indices are raised, lowered and contracted with the Minkowski metric $\eta_{\mu \nu}$. Let us split the Lagrangian into a term quadratic in the perturbations and an interaction term

$$
\mathcal{L}_{e}=\mathcal{L}_{e}^{Q}+\mathcal{L}_{e}^{(\mathrm{int})}
$$

For the quadratic term we get

$$
\mathcal{L}_{e}^{Q}=\tilde{\mathcal{L}}_{E H}^{Q}-\epsilon_{\varsigma} \frac{1}{2}(\partial \varsigma)^{2}-\epsilon_{\varphi} \frac{1}{2}(\partial \varphi)^{2}-\frac{1}{2} m_{\varsigma}^{2} \varsigma^{2},
$$

where we have defined

$$
\begin{aligned}
& \epsilon_{\varsigma} \equiv \operatorname{sign}\left(\frac{\mathcal{K}_{\sigma \sigma}^{(0)} \mathcal{K}_{\phi \phi}^{(0)}-\left(\mathcal{K}_{\sigma \phi}^{(0)}\right)^{2}}{\mathcal{K}_{\phi \phi}^{(0)}}\right), \quad \epsilon_{\varphi} \equiv \operatorname{sign}\left(\mathcal{K}_{\phi \phi}^{(0)}\right), \\
& m_{\varsigma}^{2} \equiv \epsilon_{\varsigma} \tilde{V}^{(2)} M^{2}=\epsilon_{\varsigma}\left(\frac{\mathcal{K}_{\sigma \sigma}^{(0)} \mathcal{K}_{\phi \phi}^{(0)}-\left(\mathcal{K}_{\sigma \phi}^{(0)}\right)^{2}}{\mathcal{K}_{\phi \phi}^{(0)}}\right)^{-1} V^{(2)} M^{2} .
\end{aligned}
$$

In this case, on top of the two tensorial massless degrees of freedom, the theory contains two scalar degrees of freedom among which at least one is massless. We have the following criteria for the perturbations to be well-behaved: ${ }^{16}$

- For positive definite kinetic terms (absence of ghosts):

$$
\mathcal{K}_{\sigma \sigma}^{(0)} \mathcal{K}_{\phi \phi}^{(0)}-\left(\mathcal{K}_{\sigma \phi}^{(0)}\right)^{2}>0 \text { and } \mathcal{K}_{\phi \phi}^{(0)}>0
$$

- For positive or zero mass of $\varsigma_{c}$ (absence of tachyons):

$$
V^{(2)} \geq 0
$$

\footnotetext{
${ }^{16}$ These conditions can also be formulated in a variable independent way. The first two conditions correspond to a positive definite field space metric to lowest order in the expansion around the constant background. Requiring that the matrix of second derivatives of the potential evaluated at the constant background solution should have no negative eigenvalue is the analog of the third condition.
} 
Besides, there will be phenomenological constraints coming from the coupling of the previous fields to other fields of the SM. The only remark we want to make on this respect is that the massless field $\varphi$ will be only derivatively coupled to $\sigma$ (and, moreover, by higher dimensional operators), which implies that its effects at small energies are naturally suppressed (see Section 3.2).

\subsection{Interactions and separation of scales}

We now want to consider the interactions coming from the Lagrangian (3.17) for $\Lambda_{0}=0$. In general those are represented by an infinite series of terms arising from the expansion of the functions $\tilde{\mathcal{K}}_{\phi \phi}(\tilde{\sigma})$ and $\tilde{V}(\tilde{\sigma})$ and of the metric tensor around the constant background. The interaction terms obtained from the expansion of the Ricci scalar in (3.17) are suppressed by the Planck mass. We neglect them, as we will be interested in sub-Planckian processes (we consider the cut-off of the theory to be the mass scale $M$ ). Let us consider the terms of dimension up to four:

$$
\mathcal{L}_{e}^{\text {int } \leq 4}=-\frac{1}{3 !} \kappa_{\varsigma} \varsigma^{3}-\frac{\lambda_{\varsigma}}{4 !} \varsigma^{4}-\frac{1}{4} \frac{m_{\varsigma}^{2}}{M} \varsigma^{2} \tilde{h}-\frac{1}{16} \frac{m_{\varsigma}^{2}}{M^{2}} \varsigma^{2}\left(\tilde{h}^{2}-2 \tilde{h}_{\mu \nu} \tilde{h}^{\mu \nu}\right)-\frac{1}{12} \frac{\kappa_{\varsigma}}{M} \varsigma^{3} \tilde{h}
$$

where

$$
\kappa_{\varsigma} \equiv \tilde{V}^{(3)} M, \quad \lambda_{\varsigma} \equiv \tilde{V}^{(4)}
$$

These are the relevant operators for a scalar field minimally coupled to Einstein gravity. For a generic theory (where the TDF and their derivatives are of the order of one) the mass of the field $\varsigma$ is of the order of the Planck scale (cf. (3.28)). If we want to identify the field $\varsigma$ with a low-energy degree of freedom (such as the Higgs boson of the SM), the TDF must obey several constraints. In particular, the mass of the particle must be much smaller than the mass scale $M$ (which sets the cut-off scale of the theory) :

$$
\left|\frac{m_{\varsigma}}{M}\right|=\sqrt{\left|\tilde{V}^{(2)}\right|} \ll 1
$$

This condition is similar to the fine-tuning conditions of the SM, requiring that the Fermi scale is much smaller than the Planck scale.

Besides, for the theory to be weakly coupled at energies of order $m_{\varsigma}$, we also need to have $\frac{\kappa_{\varsigma}}{m_{\varsigma}}, \lambda_{\varsigma} \lesssim 1$, which means

$$
\frac{\left|\tilde{V}^{(3)}\right|}{\sqrt{\left|\tilde{V}^{(2)}\right|}}, \tilde{V}^{(4)} \lesssim 1
$$

For the Lagrangian (3.31) to represent a consistent effective field theory at energies smaller than $M$, the corrections to it originating from the power expansions of the TDF must be suppressed (see, however, Section 3.3). The higher dimensional operators can be 
written schematically as

$$
\begin{aligned}
\mathcal{L}_{e}^{\text {int }>4}= & \sum_{n_{h}>0}^{\infty} \frac{1}{M^{n_{h}}}\left(\mathcal{L}_{e}^{Q}+\mathcal{L}_{e}^{\text {int } \leq 4}\right) \tilde{h}^{n_{h}} \\
& +\sum_{\substack{n_{h} \geq 0 \\
n_{\varsigma}>0}}^{\infty}\left(\frac{1}{M_{\phi \phi}\left(n_{h}, n_{\varsigma}\right)}\right)^{n_{h}+n_{\varsigma}}(\partial \varphi)^{2} \tilde{h}^{n_{h}} \varsigma^{n_{\varsigma}}+\sum_{\substack{n_{h} \geq 0 \\
n_{\varsigma}>4}}^{\infty}\left(\frac{1}{M_{V}\left(n_{h}, n_{\varsigma}\right)}\right)^{n_{h}+n_{\varsigma}-4} \tilde{h}^{n_{h}} \varsigma^{n_{\varsigma}},
\end{aligned}
$$

where we neglect numerical factors of order one, neglect tensor indices and define

$$
\begin{aligned}
& M_{\phi \phi}\left(n_{h}, n_{\varsigma}\right) \sim M\left|\frac{\tilde{\mathcal{K}}_{\phi \phi}^{\left(n_{\varsigma}\right)}}{\tilde{\mathcal{K}}_{\phi \phi}^{(0)}}\right|^{\frac{-1}{n_{h}+n_{\varsigma}}}, \\
& M_{V}\left(n_{h}, n_{\varsigma}\right) \sim M\left|\tilde{V}^{\left(n_{\varsigma}\right)}\right|^{\frac{-1}{n_{h}+n_{\varsigma}-4}} .
\end{aligned}
$$

The first line of (3.35) represents the standard higher dimensional operators for Einstein gravity and a minimally coupled scalar field. If the conditions (3.33) and (3.34) hold, they are all suppressed at energies below the scale $M$. The remaining terms are new higher dimensional operators that appear if the kinetic term is non-canonical and/or if the potential contains higher dimensional operators. The suppression scales of these operators are given by $M_{\phi \phi}\left(n_{h}, n_{\varsigma}\right)$ and $M_{V}\left(n_{h}, n_{\varsigma}\right)$. They are at least of the order of the Planck scale $M$ provided that

$$
\left|\frac{\tilde{\mathcal{K}}_{\phi \phi}^{\left(n_{\varsigma}\right)}}{\tilde{\mathcal{K}}_{\phi \phi}^{(0)}}\right|^{\frac{1}{n_{h}+n_{\varsigma}}} \leq 1 \quad \text { and } \quad\left|\tilde{V}^{\left(n_{\varsigma}\right)}\right|^{\frac{1}{n_{h}+n_{\varsigma}-4}} \leq 1
$$

Let us now summarize the findings of this section. We have considered a scale-invariant theory of a scalar field coupled to TDiff gravity, which is described by the action (3.7) (or equivalently (3.17)). If there exists a value of $\sigma_{0}$ for which $v\left(\sigma_{0}\right)=v^{\prime}\left(\sigma_{0}\right)=0$ (i.e. $\tilde{V}^{(0)}=\tilde{V}^{(1)}=0$ ), there exists a family of maximally symmetric solutions of the equations of motion, corresponding to flat space-time and constant scalar fields. Those solutions for which $\phi_{0} \neq 0$ spontaneously break the dilatation symmetry of the theory. Besides, scale invariance can be independently broken by an integration constant $\Lambda_{0}$, which introduces a run-away potential for the dilaton field. The quadratic analysis of perturbations around the background solutions with $\Lambda_{0}=0$ shows that if the conditions $\mathcal{K}_{\sigma \sigma}^{(0)} \mathcal{K}_{\phi \phi}^{(0)}-\left(\mathcal{K}_{\sigma \phi}^{(0)}\right)^{2}>0$ and $\tilde{\mathcal{K}}_{\phi \phi}^{(0)}>0$ are satisfied, the theory describes two massless tensor degrees of freedom, a massless scalar and a scalar of mass $m_{\varsigma}^{2}=\tilde{V}^{(2)}$. The scale $M$ for gravity and the scales $m_{\varsigma}$ and $\kappa_{\varsigma}$ associated to the scalar field are induced by the non-zero value of $\phi_{0} .{ }^{17}$ If the theory-defining functions are such that the conditions (3.33), (3.34) and (3.37) are fulfilled,

\footnotetext{
${ }^{17}$ This fact is easier to see in the J-frame. Expanding around the constant background one finds that the coupling constant of the tensor modes as well as the mass of the scalar mode are proportional to $\phi_{0}$. In the E-frame this fact is implicit, since the transformation to the E-frame is only allowed if $\phi_{0} \neq 0$.
} 
the scalar and the tensor sectors decouple and all the non-renormalizable interactions are suppressed below the scale $M$. In this case, at energies well below $M$, the scalar field phenomenology resulting from the theory (3.7) is indistinguishable from the phenomenology of the corresponding renormalizable scalar-field theory.

\subsection{Dependence on the choice of variables and exact renormalizability}

Under very general assumptions, the Lagrangian (3.7) can be brought to the form (3.17) by a non-singular change of variables. Furthermore, one may still perform field redefinitions of the form $(\tilde{\sigma}, \tilde{\phi}) \mapsto\left(\tilde{\sigma}^{\prime}, \tilde{\phi}^{\prime}\right)$ that modify the explicit expressions of the functions $\tilde{\mathcal{K}}_{\phi \phi}(\tilde{\sigma})$, $\tilde{V}(\tilde{\sigma})$, etc. For example, for some functions $\tilde{\mathcal{K}}_{\phi \phi}(\tilde{\sigma})$ one can make a change of variables which brings the kinetic term to the canonical form (see below). Also the functions $\tilde{V}(\tilde{\sigma})$ and $\tilde{\mathcal{K}}_{\Lambda_{0}}(\tilde{\sigma})$ appearing in the potential take different forms for different choices of variables. For instance, there might exist variables in terms of which the potential is polynomial, whereas in another set it contains exponential functions.

In the previous sections we expanded the Lagrangian around the constant background (3.24). The idea is that perturbations around this background can be quantized and interpreted as particles. Their tree-level masses and coupling constants are given by the coefficients of the Taylor expansion around the point $\tilde{\sigma}=0$, i.e. by terms of the form $\tilde{\mathcal{K}}_{\phi \phi}^{(n)}$ and $\tilde{V}^{(n)}$. Certainly, since the functions depend on the variable choice, for different sets of variables, tree-masses and coupling constants will take different values. Nevertheless, the equivalence theorems of [39] show that the so constructed quantum theories are equivalent for all choices of variables. A consequence of these theorems is that whenever one takes into account the whole (possibly infinite) series of terms in the Lagrangian to compute $S$-matrix elements, the result will not depend on the choice of variables if the transformations are well-defined perturbatively. The situations is different, however, if one uses effective field theory arguments to truncate the Lagrangian because, as already mentioned, the individual terms of the series expansions do depend on the choice of variables. This means that conditions like (3.33), (3.34) and (3.37) depend on the choice of variables. Therefore, applied to arbitrary variables, such conditions should be considered as sufficient but not necessary. It can happen, for instance, that for some choice of variables some of the suppression conditions (3.37) do not hold, but that the corresponding terms are nevertheless irrelevant ${ }^{18}$. In order to have a variable independent statement, the ensemble of conditions (3.33), (3.34) and (3.37) should be read as follows:

"If there exists a set of variables in terms of which the conditions (3.33), (3.34) and (3.37) hold, then, at energies well below $M$, the scalar-field theory contained in (3.7) is indistinguishable from the corresponding renormalizable theory."

Understood this way, the conditions are necessary and sufficient.

As a particular example of the previous reasoning, one may wonder whether there exists a set of field variables in terms of which the kinetic part of the Lagrangian 3.17)

\footnotetext{
${ }^{18}$ Technically, this can happen in the following way. The interaction Lagrangian can contain terms with big coefficients. These terms violate some of the conditions (3.37) and are therefore expected to be important much below the scale $M$. However, there can be cancellations between terms of the different series contained in (3.35) which make that also terms that violate the conditions (3.37) can be irrelevant.
} 
takes exactly the canonical form. The condition for such variables to exist is the vanishing of the Riemann tensor computed from the field space metric [40]

$$
\left\{\mathcal{K}_{i j}(\tilde{\sigma}, \tilde{\phi})\right\}=\left(\begin{array}{cc}
\epsilon_{\sigma} M^{2} & 0 \\
0 & \tilde{\mathcal{K}}_{\phi \phi}(\tilde{\sigma})
\end{array}\right)
$$

This condition corresponds to ${ }^{19}$

$$
\tilde{\mathcal{K}}_{\phi \phi}^{\prime}(\tilde{\sigma})^{2}-2 \tilde{\mathcal{K}}_{\phi \phi}(\tilde{\sigma}) \tilde{\mathcal{K}}_{\phi \phi}^{\prime \prime}(\tilde{\sigma})=0
$$

Functions $\tilde{\mathcal{K}}_{\phi \phi}(\tilde{\sigma})$ which satisfy this equation have the form

$$
\tilde{\mathcal{K}}_{\phi \phi}(\tilde{\sigma})=\left(c_{1} \tilde{\sigma}+c_{2}\right)^{2}
$$

where $c_{1}$ and $c_{2}$ are arbitrary constants. One can also formulate the conditions which guarantee that for the variables that give a canonical kinetic term, the scalar field potential (for $\Lambda_{0}=0$ ) becomes a polynomial of a maximum order $p$,

$$
\tilde{V}(\tilde{\sigma})_{; i_{1} ; i_{2} ; i_{3} ; \ldots ; i_{p+1}}=0
$$

where the semicolon stands for the covariant derivative with respect to the metric (3.38). If these conditions hold for $p=4$ and at the same time condition (3.39) is fulfilled, the scalar part of the Lagrangian describes a tree unitary and renormalizable quantum field theory [40]. For this to be the case, the function $\tilde{V}(\tilde{\sigma})$ has to be of the form

$$
\begin{array}{lrl}
\tilde{V}(\tilde{\sigma})=v_{0}+v_{1} \tilde{\sigma}+v_{2} \tilde{\sigma}^{2}+v_{3} \tilde{\sigma}^{3}+v_{4} \tilde{\sigma}^{4}, & \text { if } & c_{1}=0, \\
\tilde{V}(\tilde{\sigma})=v_{0}+\tilde{\sigma}\left(\tilde{\sigma}+2 c_{2} / c_{1}\right)\left(v_{4} \tilde{\sigma}^{2}+2 c_{2} v_{4} / c_{1} \tilde{\sigma}+v_{2}-4 c_{2}^{2} v_{4} / c_{1}^{2}\right), & \text { if } \quad & c_{1} \neq 0,
\end{array}
$$

where $v_{0}, v_{1}, v_{2}, v_{3}$ and $v_{4}$ are arbitrary constants. If we also impose the conditions (3.23), which correspond to $\tilde{V}(0)=\tilde{V}^{\prime}(0)=0$, we can further restrict the form of the function $\tilde{V}(\tilde{\sigma})$ to

$$
\begin{aligned}
& \tilde{V}(\tilde{\sigma})=v_{2} \tilde{\sigma}^{2}+v_{3} \tilde{\sigma}^{3}+v_{4} \tilde{\sigma}^{4}, \quad \text { if } \quad c_{1}=0, \\
& \tilde{V}(\tilde{\sigma})=\tilde{\sigma}^{2}\left(v_{2}+v_{4} \tilde{\sigma}^{2}\right), \\
& \tilde{V}(\tilde{\sigma})=v_{4} \tilde{\sigma}^{2}\left(\tilde{\sigma}+2 c_{2} / c_{1}\right)^{2}, \quad \text { if } \quad c_{1} \neq 0 \text { and } c_{2}=0,
\end{aligned}
$$

\footnotetext{
${ }^{19}$ In terms of the functions without tilde, the same condition reads $\mathcal{K}_{\phi \phi}^{\prime}(\sigma)\left(\mathcal{K}_{\phi \phi}(\sigma) \mathcal{K}_{\sigma \sigma}^{\prime}(\sigma)+\mathcal{K}_{\phi \phi}^{\prime}(\sigma) \mathcal{K}_{\sigma \sigma}(\sigma)-2 \mathcal{K}_{\sigma \phi}(\sigma) \mathcal{K}_{\sigma \phi}^{\prime}(\sigma)\right)+2\left(\mathcal{K}_{\sigma \phi}(\sigma)^{2}-\mathcal{K}_{\phi \phi}(\sigma) \mathcal{K}_{\sigma \sigma}(\sigma)\right) \mathcal{K}_{\phi \phi}^{\prime \prime}(\sigma)=0$.
} 


\section{Including gauge bosons}

In this section we will consider the addition of (massive) gauge fields to the previous picture of scale-invariant TDiff Lagrangians. Remind that in the Higgs mechanism, gauge fields get their masses from a non-zero expectation value of a scalar field. We are going to show how a similar phenomenon can occur due to spontaneous breaking of scale invariance in a scale-invariant TDiff theory, where the massive field $\sigma$ will play a role similar to the Higgs field of the SM. For simplicity we will consider the case of an Abelian gauge group.

If the scalar field $\phi$ is promoted to a complex field, the Lagrangian (3.7) is invariant under a global $U(1)$ symmetry. This symmetry can be turned into a gauge symmetry by introducing an Abelian gauge field (note that gauge fields have scaling dimension $d_{A}=1$ ). The generalization of $(3.7)$ to this case reads

$$
\begin{aligned}
\frac{\mathcal{L}}{\sqrt{-g}}= & -\frac{1}{2}|\phi|^{2} f(-g) R-\frac{1}{2}|\phi|^{2} \mathcal{G}_{g g}(-g)(\partial g)^{2}-\frac{1}{2} \mathcal{G}_{\phi \phi}(-g) D \phi \cdot(D \phi)^{*} \\
& +\frac{1}{2} \mathcal{G}_{g \phi}^{*}(-g) \phi^{*} \partial g \cdot D \phi+\frac{1}{2} \mathcal{G}_{g \phi}(-g) \phi \partial g \cdot(D \phi)^{*}-\frac{1}{2} \mathcal{G}_{n a}(-g) \partial|\phi| \cdot \partial|\phi| \\
& -v(-g)\left|\phi \phi^{*}\right|^{2}-\frac{1}{4} \mathcal{G}_{A A}(-g) F^{2}-\frac{1}{4} \mathcal{G}_{\varepsilon}(-g) F \wedge F,
\end{aligned}
$$

where the covariant derivative is defined as $D_{\mu} \equiv \partial_{\mu}-\mathrm{i} e A_{\mu}$ and the function $\mathcal{G}_{g \phi}(-g)$ is complex valued. In this action we have also included the non-analytical term $\partial|\phi|$. Notice that this term is unique and perfectly well defined around the background $\phi_{0} \neq 0$, so it is natural to consider it as a term in the potential on the same footing as we consider generic $\mathrm{TDF}^{20}$. Moreover, we have defined the wedge product as $F \wedge F=\epsilon^{\mu \nu \rho \sigma} F_{\mu \nu} F_{\rho \sigma}$, where $\epsilon_{\mu \nu \rho \sigma} \equiv \sqrt{-g} \varepsilon_{\mu \nu \rho \sigma}$, with $\varepsilon_{\mu \nu \rho \sigma}$ being the standard Levi-Civita tensor. We will analyze the theory in the unitary gauge $\phi^{*}=\phi$, in which the Lagrangian reads

$$
\begin{aligned}
\frac{\mathcal{L}}{\sqrt{-g}}= & -\frac{1}{2} \phi^{2} f(-g) R-\frac{1}{2} \phi^{2} \mathcal{G}_{g g}(-g)(\partial g)^{2}-\frac{1}{2}\left(\mathcal{G}_{\phi \phi}(-g)+\mathcal{G}_{n a}(-g)\right)(\partial \phi)^{2} \\
& +\operatorname{Re}\left[\mathcal{G}_{g \phi}(-g)\right] \phi \partial g \cdot \partial \phi+e \operatorname{Im}\left[\mathcal{G}_{g \phi}(-g)\right] \phi^{2} \partial g \cdot A-\frac{1}{2} e^{2} \mathcal{G}_{\phi \phi}(-g) A^{2} \phi^{2} \\
& -v(-g) \phi^{4}-\frac{1}{4} \mathcal{G}_{A A}(-g) F^{2}-\frac{1}{4} \mathcal{G}_{\varepsilon}(-g) F \wedge F
\end{aligned}
$$

where Re and Im stand for the real and imaginary part, respectively. Following the formalism developed in Section 2.1, one can directly write down the equivalent Diff invariant

\footnotetext{
${ }^{20}$ Certainly, around the symmetry breaking background those terms involve non-renormalizable operators. Considering the perturbation theory for certain TDF, those operators should be placed beyond the cut-off of the theory which basically implies that the non-analytical term should be suppressed altogether. However, as we emphasized in Section 3.3, this conclusion depends on the choice of fields and certain higher order operators in one representation may be resummed to a renormalizable form by a local field redefinition.
} 
theory in the Einstein frame as (see (3.11))

$$
\begin{aligned}
\frac{\mathcal{L}_{e}}{\sqrt{-\tilde{g}}}= & -\frac{1}{2} M^{2} \tilde{R}-\frac{1}{2} M^{2} \mathcal{K}_{\sigma \sigma}(\sigma)(\partial \sigma)^{2}-\frac{1}{2} M^{2} \mathcal{K}_{\phi \phi}(\sigma)(\partial \ln (\phi / M))^{2} \\
& -M^{2} \mathcal{K}_{\sigma \phi}(\sigma) \partial \sigma \cdot \partial \ln (\phi / M)-e M^{2} \mathcal{K}_{\sigma A}(\sigma) \partial \sigma \cdot A-\frac{1}{2} e^{2} M^{2} \mathcal{K}_{i n t}(\sigma) A^{2} \\
& -M^{4} V(\sigma)-\frac{1}{4} \mathcal{K}_{A A}(\sigma) F^{2}-\frac{1}{4} \mathcal{K}_{\varepsilon}(\sigma) F \wedge F-\frac{M^{4} \Lambda_{0}}{\phi^{4} f(\sigma)^{2} \sqrt{\sigma}}
\end{aligned}
$$

where

$$
\begin{array}{rlrl}
\mathcal{K}_{\sigma \sigma}(\sigma) & =\frac{\mathcal{G}_{g g}(\sigma)}{f(\sigma)}+\frac{3}{2}\left(\frac{f^{\prime}(\sigma)}{f(\sigma)}\right)^{2}, & \mathcal{K}_{\phi \phi}(\sigma) & =\frac{\mathcal{G}_{\phi \phi}(\sigma)+\mathcal{G}_{n a}(\sigma)}{f(\sigma)}+6, \\
\mathcal{K}_{\sigma \phi}(\sigma)=\frac{\operatorname{Re}\left[\mathcal{G}_{g \phi}(\sigma)\right]}{f(\sigma)}+3 \frac{f^{\prime}(\sigma)}{f(\sigma)}, & \mathcal{K}_{\sigma A}(\sigma) & =\frac{\operatorname{Im}\left[\mathcal{G}_{g \phi}(\sigma)\right]}{f(\sigma)}, \\
\mathcal{K}_{i n t}(\sigma)=\frac{\mathcal{G}_{\phi \phi}(\sigma)}{f(\sigma)}, & V(\sigma) & =\frac{v(\sigma)}{f(\sigma)^{2}}, \\
\mathcal{K}_{A A}(\sigma) & =\mathcal{G}_{A A}(\sigma), & \mathcal{K}_{\varepsilon}(\sigma) & =\mathcal{G}_{\varepsilon}(\sigma) .
\end{array}
$$

At this point, as in the case without gauge fields, we can make a field redefinition in order to eliminate the derivative couplings between the different fields. This will simplify the interpretation of the theory as a description of interacting particles. The extension of expression (3.16) to this case is ${ }^{21}$

$$
\begin{gathered}
\tilde{\sigma}=\int_{\sigma_{0}}^{\sigma} \mathrm{d} \sigma^{\prime} \sqrt{\left|\frac{\mathcal{K}_{\sigma \sigma} \mathcal{K}_{\phi \phi}-\mathcal{K}_{\sigma \phi}^{2}}{\mathcal{K}_{\phi \phi}}-\frac{\mathcal{K}_{\sigma A}^{2}}{\mathcal{K}_{i n t}}\right|}, \quad \tilde{\phi}=M\left(\ln \frac{\phi}{M}+\int_{\sigma_{0}}^{\sigma} \mathrm{d} \sigma^{\prime} \frac{\mathcal{K}_{\sigma \phi}}{\mathcal{K}_{\phi \phi}}\right), \\
\tilde{A}_{\mu}=A_{\mu}+\frac{1}{e} \frac{\mathcal{K}_{\sigma A}}{\mathcal{K}_{i n t}} \partial_{\mu} \sigma,
\end{gathered}
$$

in terms of which the above Lagrangian reads

$$
\begin{aligned}
\frac{\mathcal{L}_{e}}{\sqrt{-\tilde{g}}}= & -\frac{1}{2} M^{2} \tilde{R}-\frac{1}{2} \epsilon_{\sigma} M^{2}(\partial \tilde{\sigma})^{2}-\frac{1}{2} \tilde{\mathcal{K}}_{\phi \phi}(\tilde{\sigma})(\partial \tilde{\phi})^{2} \\
& -\frac{1}{2} e^{2} \tilde{\mathcal{K}}_{i n t}(\tilde{\sigma}) M^{2} \tilde{A}^{2}-\frac{1}{4} \tilde{\mathcal{K}}_{A A}(\tilde{\sigma}) \tilde{F}^{2}-\frac{1}{4} \tilde{\mathcal{K}}_{\varepsilon}(\tilde{\sigma}) \tilde{F} \wedge \tilde{F} \\
& -\tilde{V}(\tilde{\sigma}) M^{4}-\Lambda_{0} \tilde{\mathcal{K}}_{\Lambda_{0}} \exp \left(-\frac{4 \tilde{\phi}}{M}\right),
\end{aligned}
$$

where $\epsilon_{\sigma}=\operatorname{sign}\left(\frac{\mathcal{K}_{\sigma \sigma} \mathcal{K}_{\phi \phi}-\mathcal{K}_{\sigma \phi}^{2}}{\mathcal{K}_{\phi \phi}}-\frac{\mathcal{K}_{\sigma A}^{2}}{\mathcal{K}_{i n t}}\right)$ and $\tilde{\mathcal{K}}_{\Lambda_{0}}(\tilde{\sigma})$ is defined in (3.18). Note that the field $\tilde{\phi}$ is completely decoupled from the vector fields (which follows from scale and gauge invariance), thus the mass of the vector bosons is related to the interaction with the "gravitational" field $\tilde{\sigma}$. In this loose sense, the role of the Higgs field is played by the determinant of the metric ${ }^{22}$. The previous Lagrangian may be subject to different constraints that we will consider in the next sections.

\footnotetext{
${ }^{21}$ We assume that $\mathcal{K}_{\phi \phi}, \mathcal{K}_{\text {int }}$ and $\frac{\mathcal{K}_{\sigma \sigma} \mathcal{K}_{\phi \phi}-\mathcal{K}_{\sigma \phi}^{2}}{\mathcal{K}_{\phi \phi}}-\frac{\mathcal{K}_{\sigma A}^{2}}{\mathcal{K}_{i n t}}$ are non-vanishing.

${ }^{22} \mathrm{~A}$ possible connection between the Higgs field and the determinant of the metric was suggested previously in 41, 42] from different considerations.
} 


\subsection{Local degrees of freedom}

Like in the case without gauge fields, the existence of a constant solution $\tilde{g}_{\mu \nu}=\eta_{\mu \nu}, \tilde{\sigma}=0$, $\tilde{\phi}=\tilde{\phi}_{0}$ and $\tilde{A}_{\mu}=0$ is assured by the conditions (we also assume $f\left(\sigma_{0}\right) \neq 0$ )

$$
v\left(\sigma_{0}\right)=v^{\prime}\left(\sigma_{0}\right)=0 .
$$

Let us also recall that the constant solution has $\Lambda_{0}=0$. We again want to examine the nature of the perturbations around the constant solution, which we define as

$$
\begin{array}{ll}
\tilde{g}_{\mu \nu}=\eta_{\mu \nu}+\frac{\tilde{h}_{\mu \nu}}{M}, \quad \tilde{\sigma}=\frac{\varsigma}{M}, \\
\tilde{\phi}=\tilde{\phi}_{0}+\frac{\varphi}{\left|\tilde{\mathcal{K}}_{\phi \phi}^{(0)}\right|^{1 / 2}}, \quad \tilde{A}_{\mu}=\frac{\tilde{A}_{\mu}^{c}}{\left|\tilde{\mathcal{K}}_{A A}^{(0)}\right|^{1 / 2}} .
\end{array}
$$

In the rest of Section 14, Lorentz indices are raised, lowered and contracted with the Minkowski metric $\eta_{\mu \nu}$. To quadratic order the Lagrangian (4.6) reduces to

$$
\mathcal{L}_{e}^{Q}=\tilde{\mathcal{L}}_{E H}^{Q}-\epsilon_{\varsigma} \frac{1}{2}(\partial \varsigma)^{2}-\epsilon_{\varphi} \frac{1}{2}(\partial \varphi)^{2}-\frac{1}{2} m_{\varsigma}^{2} \varsigma^{2}-\epsilon_{A} \frac{1}{4} \tilde{F}^{2}-\frac{1}{2} m_{A}^{2} \tilde{A}^{2}
$$

where

$$
\begin{gathered}
\epsilon_{\varsigma} \equiv \operatorname{sign}\left(\frac{\mathcal{K}_{\sigma \sigma}^{(0)} \mathcal{K}_{\phi \phi}^{(0)}-\left(\mathcal{K}_{\sigma \phi}^{(0)}\right)^{2}}{\mathcal{K}_{\phi \phi}^{(0)}}-\frac{\left(\mathcal{K}_{\sigma A}^{(0)}\right)^{2}}{\mathcal{K}_{\text {int }}^{(0)}}\right), \quad \epsilon_{\varphi} \equiv \operatorname{sign}\left(\mathcal{K}_{\phi \phi}^{(0)}\right), \epsilon_{A} \equiv \operatorname{sign}\left(\mathcal{K}_{A A}^{(0)}\right), \\
m_{A}^{2} \equiv \epsilon_{A} e^{2} \frac{\mathcal{K}_{i n t}^{(0)}}{\mathcal{K}_{A A}^{(0)}} M^{2}, \\
m_{\varsigma}^{2} \equiv \epsilon_{\varsigma} \tilde{V}^{(2)} M^{2}=\epsilon_{\varsigma}\left(\frac{\mathcal{K}_{\sigma \sigma}^{(0)} \mathcal{K}_{\phi \phi}^{(0)}-\left(\mathcal{K}_{\sigma \phi}^{(0)}\right)^{2}}{\mathcal{K}_{\phi \phi}^{(0)}}-\frac{\left(\mathcal{K}_{\sigma A}^{(0)}\right)^{2}}{\mathcal{K}_{\text {int }}^{(0)}}\right)^{-1} V^{(2)} M^{2} .
\end{gathered}
$$

At the level of the quadratic Lagrangian, the following conditions must be satisfied:

- For positive definite kinetic terms (absence of ghosts):

$$
\epsilon_{\varsigma}, \epsilon_{\varphi}, \epsilon_{A}=1
$$

- For positive or zero masses (absence of tachyons):

$$
m_{\varsigma}^{2}, m_{A}^{2} \geq 0 \text {. }
$$




\subsection{Interactions and separation of scales}

The terms of dimension up to four are

$$
\begin{aligned}
\mathcal{L}_{e}^{\text {int } \leq 4}= & -\frac{1}{3 !} \kappa_{\varsigma} \varsigma^{3}-\frac{\lambda_{\varsigma}}{4 !} \varsigma^{4}-\frac{1}{2} \kappa_{A} \eta^{\mu \nu} \tilde{A}_{\mu}^{c} \tilde{A}_{\nu}^{c} \varsigma-\frac{1}{4} \lambda_{A} \eta^{\mu \nu} \tilde{A}_{\mu}^{c} \tilde{A}_{\nu}^{c} \varsigma^{2} \\
& -\frac{1}{4} \frac{m_{\varsigma}^{2}}{M} \varsigma^{2} \tilde{h}-\frac{1}{16} \frac{m_{\varsigma}^{2}}{M^{2}} \varsigma^{2}\left(\tilde{h}^{2}-2 \tilde{h}_{\mu \nu} \tilde{h}^{\mu \nu}\right)-\frac{1}{12} \frac{\kappa_{\varsigma}}{M} \varsigma^{3} \tilde{h} \\
& -\frac{1}{4} \frac{m_{A}^{2}}{M} \tilde{A}_{\mu}^{c} \tilde{A}_{\nu}^{c}\left(\eta^{\mu \nu} \tilde{h}-2 \tilde{h}^{\mu \nu}\right)-\frac{1}{4} \frac{\kappa_{A}}{M} \varsigma \tilde{A}_{\mu}^{c} \tilde{A}_{\nu}^{c}\left(\eta^{\mu \nu} \tilde{h}-2 \tilde{h}^{\mu \nu}\right) \\
& -\frac{1}{16} \frac{m_{A}^{2}}{M^{2}} \tilde{A}_{\mu}^{c} \tilde{A}_{\nu}^{c}\left(\eta^{\mu \nu} \tilde{h}^{2}-4 \tilde{h}^{\mu \nu} \tilde{h}^{2}-2 \eta^{\mu \nu} \tilde{h}_{\rho \sigma} \tilde{h}^{\rho \sigma}+8 \tilde{h}_{\rho}^{\mu} \tilde{h}^{\rho \nu}\right)
\end{aligned}
$$

where we have defined the parameters

$$
\kappa_{\varsigma} \equiv \tilde{V}^{(3)} M, \quad \lambda_{\varsigma} \equiv \tilde{V}^{(4)}, \quad \kappa_{A} \equiv e^{2} \frac{\tilde{\mathcal{K}}_{i n t}^{(1)}}{\tilde{\mathcal{K}}_{A A}^{(0)}} M, \quad \lambda_{A} \equiv e^{2} \frac{\tilde{\mathcal{K}}_{i n t}^{(2)}}{\tilde{\mathcal{K}}_{A A}^{(0)}} .
$$

As in the previous section, we will require that the mass scales of the fields $\varsigma$ and $\tilde{A}_{\mu}$ are parametrically smaller than the cut-off $M$,

$$
\left|\frac{m_{\varsigma}}{M}\right|=\sqrt{\left|\tilde{V}^{(2)}\right|} \ll 1, \quad\left|\frac{m_{A}}{M}\right|=\sqrt{\left|\tilde{e}^{2} \mathcal{K}_{\text {int }}^{(0)}\right|} \ll 1,
$$

with the definition $\tilde{e}^{2} \equiv \frac{e^{2}}{\mathcal{K}_{A A}^{(0)}}$. In addition, we have the following conditions that prevent the theory from being strongly coupled,

$$
\left\{\frac{\kappa_{\varsigma}}{m_{\min }}, \frac{\kappa_{A}}{m_{\min }}, \lambda_{\varsigma}, \lambda_{A}, \lesssim 1\right.
$$

where $m_{\min } \equiv \min \left(m_{\varsigma}, m_{A}\right)$. Note that the first two conditions might not be necessary for a particular structure of the interactions. In particular, these conditions are not necessary if the theory corresponds to the Abelian Higgs model.

The higher dimensional terms can be written schematically as

$$
\begin{aligned}
\mathcal{L}_{e}^{\text {int }>4}= & \sum_{n_{h}>0}^{\infty} \frac{1}{M^{n_{h}}}\left(\mathcal{L}_{e}^{Q}+\mathcal{L}_{e}^{\text {int } \leq 4}\right) \tilde{h}^{n_{h}} \\
& +\sum_{\substack{n_{h} \geq 0 \\
n_{\varsigma}>0}}^{\infty}\left(\frac{1}{M_{\phi \phi}\left(n_{h}, n_{\varsigma}\right)}\right)^{n_{h}+n_{\varsigma}}(\partial \varphi)^{2} \tilde{h}^{n_{h}} \varsigma^{n_{\varsigma}}+\sum_{\substack{n_{h} \geq 0 \\
n_{\varsigma}>4}}^{\infty}\left(\frac{1}{M_{V}\left(n_{h}, n_{\varsigma}\right)}\right)^{n_{h}+n_{\varsigma}-4} \tilde{h}^{n_{h}} \varsigma^{n_{\varsigma}} \\
& +\sum_{\substack{n_{h} \geq 0 \\
n_{\varsigma}>2}}^{\infty}\left(\frac{1}{M_{i n t}\left(n_{h}, h_{\varsigma}\right)}\right)^{n_{h}+n_{\varsigma}-2}\left(\tilde{A}^{c}\right)^{2} \tilde{h}^{n_{h}} \varsigma^{n_{\varsigma}} \\
& +\left(\sum_{\substack{n_{h} \geq 0 \\
n_{\varsigma}>0}}^{\infty}\left(\frac{1}{M_{A A}\left(n_{h}, n_{\varsigma}\right)}\right)^{n_{h}+n_{\varsigma}}+\sum_{\substack{n_{h} \geq 0 \\
n_{\varsigma}>0}}^{\infty}\left(\frac{1}{M_{\varepsilon}\left(n_{h}, n_{\varsigma}\right)}\right)^{n_{h}+n_{\varsigma}}\right) \partial^{2}\left(\tilde{A}^{c}\right)^{2} \tilde{h}^{n_{h}} \varsigma^{n_{\varsigma}},
\end{aligned}
$$


where, as before, we neglect numerical factors of order one, neglect tensor indices and define the suppression scales

$$
\begin{aligned}
& M_{\phi \phi}\left(n_{h}, n_{\varsigma}\right) \sim M\left|\frac{\tilde{\mathcal{K}}_{\phi \phi}^{\left(n_{\varsigma}\right)}}{\tilde{\mathcal{K}}_{\phi \phi}^{(0)}}\right|^{\frac{-1}{n_{h}+n_{\varsigma}}}, \quad M_{V}\left(n_{h}, n_{\varsigma}\right) \sim M\left|\tilde{V}^{\left(n_{\varsigma}\right)}\right|^{\frac{-1}{n_{h}+n_{\varsigma}-4}}, \\
& M_{i n t}\left(n_{h}, n_{\varsigma}\right) \sim M\left|\tilde{e}^{2} \tilde{\mathcal{K}}_{i n t}^{\left(n_{\varsigma}\right)}\right|^{\frac{-1}{n_{h}+n_{\varsigma}}}, \quad M_{A A}\left(n_{h}, n_{\varsigma}\right) \sim M\left|\frac{\tilde{\mathcal{K}}_{A A}^{\left(n_{\varsigma}\right)}}{\tilde{\mathcal{K}}_{A A}^{(0)}}\right|^{\frac{-1}{n_{h}+n_{\varsigma}}}, \\
& M_{\varepsilon}\left(n_{h}, n_{\varsigma}\right) \sim M\left|\frac{\tilde{\mathcal{K}}_{\varepsilon}^{\left(n_{\varsigma}\right)}}{\tilde{\mathcal{K}}_{A A}^{(0)}}\right|^{\frac{-1}{n_{h}+n_{\varsigma}}} .
\end{aligned}
$$

The first term in (4.17) represents the standard higher dimensional operators of a theory minimally coupled to gravity, and are suppressed at energies below $M$ as soon as the conditions (4.15) and (4.16) hold. The additional operators come with the suppression scales $M_{\phi \phi}\left(n_{h}, n_{\varsigma}\right), M_{V}\left(n_{h}, n_{\varsigma}\right), M_{i n t}\left(n_{h}, n_{\varsigma}\right), M_{A A}\left(n_{h}, n_{\varsigma}\right)$ and $M_{\varepsilon}\left(n_{h}, n_{\varsigma}\right)$. These are comparable to or bigger than the scale $M$ whenever

$$
\begin{aligned}
& \left|\frac{\tilde{\mathcal{K}}_{\phi \phi}^{\left(n_{\varsigma}\right)}}{\tilde{\mathcal{K}}_{\phi \phi}^{(0)}}\right|^{\frac{1}{n_{h}+n_{\varsigma}}} \leq 1, \quad\left|\tilde{V}^{\left(n_{\varsigma}\right)}\right|^{\frac{1}{n_{h}+n_{\varsigma}-4}} \leq 1, \quad\left|\tilde{e}^{2} \tilde{\mathcal{K}}_{i n t}^{\left(n_{\varsigma}\right)}\right|^{\frac{1}{n_{h}+n_{\varsigma}}} \leq 1, \\
& \left|\frac{\tilde{\mathcal{K}}_{A A}^{\left(n_{\varsigma}\right)}}{\tilde{\mathcal{K}}_{A A}^{(0)}}\right|^{\frac{1}{n_{h}+n_{\varsigma}}} \leq 1, \quad\left|\frac{\tilde{\mathcal{K}}_{\varepsilon}^{\left(n_{\varsigma}\right)}}{\tilde{\mathcal{K}}_{A A}^{(0)}}\right|^{\frac{1}{n_{h}+n_{\varsigma}}} \leq 1
\end{aligned}
$$

for all values $n_{\varsigma}$ and $n_{h}$ can take in the sums in (4.17). If the conditions (4.11), (4.12), (4.15), (4.16), (4.16) and (4.19) are met, the effective Lagrangian for describing the scalar and vector sectors at energies far below $M$ is

$$
\begin{aligned}
\mathcal{L}_{e}^{\mathrm{eff}}= & -\frac{1}{2}(\partial \varsigma)^{2}-\frac{1}{2}(\partial \varphi)^{2}-\frac{1}{2} m_{\varsigma}^{2} \varsigma^{2}-\frac{1}{3 !} \kappa_{\varsigma} \varsigma^{3}-\frac{\lambda_{\varsigma}}{4 !} \varsigma^{4} \\
& -\frac{1}{4}\left(\tilde{F}^{c}\right)^{2}-\frac{1}{2} m_{A}^{2}\left(\tilde{A}^{c}\right)^{2}-\frac{1}{2} \kappa_{A}\left(\tilde{A}^{c}\right)^{2} \varsigma-\frac{1}{4} \lambda_{A}\left(\tilde{A}^{c}\right)^{2} \varsigma^{2} .
\end{aligned}
$$

We would like this Lagrangian to give rise to a consistent quantum field theory at energies low with respect to $M$. It has been shown $[40$ that the only tree-unitary theories containing scalar fields and massive vector particles are those that correspond to a spontaneously broken gauge theory ${ }^{23}$. Thus, for our model to be tree-unitary at energies below $M$ (and above $m_{A}$ ), the above effective Lagrangian should correspond to the Abelian Higgs model in the unitary gauge. This means that the six couplings $m_{\varsigma}, \kappa_{\varsigma}, \lambda_{\varsigma}, m_{A}, \kappa_{A}$ and $\lambda_{A}$ should satisfy the three relations

$$
\frac{\lambda_{\varsigma}}{\lambda_{A}}=\frac{\kappa_{\varsigma}}{\kappa_{A}}, \quad \frac{\lambda_{\varsigma}}{\lambda_{A}}=\frac{3}{2} \frac{m_{\varsigma}^{2}}{m_{A}^{2}}, \quad m_{\varsigma}^{2}=\frac{1}{3} \frac{\kappa_{\varsigma}^{2}}{\lambda_{\varsigma}} .
$$

\footnotetext{
${ }^{23}$ For theories with a conserved current, like in fermionic theories, the models where Abelian massive fields interact just with the conserved current are also allowed 40] .
} 
In the present model, these relations can be translated to the following conditions on the TDF:

$$
\frac{\tilde{\mathcal{K}}_{i n t}^{(0)}}{\tilde{\mathcal{K}}_{i n t}^{(1)}} \simeq \frac{1}{2} \frac{\tilde{\mathcal{K}}_{i n t}^{(1)}}{\tilde{\mathcal{K}}_{i n t}^{(2)}}, \quad \frac{\tilde{V}^{(2)}}{\tilde{V}^{(3)}} \simeq \frac{2}{3} \frac{\tilde{\mathcal{K}}_{i n t}^{(0)}}{\tilde{\mathcal{K}}_{i n t}^{(1)}}, \quad \frac{\tilde{V}^{(2)}}{\tilde{V}^{(3)}} \simeq \frac{1}{3} \frac{\tilde{V}^{(3)}}{\tilde{V}^{(4)}}
$$

where by the approximate equalities we mean that the relation should hold up to suppressed terms, i.e. for two quantities $a$ and $b$ one has $a \simeq b$ whenever $a=b\left(1+\mathcal{O}\left(\frac{m_{\varsigma}}{M}, \frac{\kappa_{\varsigma}}{M}, \frac{m_{A}}{M}, \frac{\kappa_{A}}{M}\right)\right)$. These conditions are expected to be stable under radiative corrections since they approximately correspond to a gauge theory with spontaneous symmetry breaking.

We can now draw the following conclusion. If there exists a set of variables in terms of which the conditions (4.7), 4.11), (4.12), (4.15), 4.16), (4.19) and (4.22) hold, then at energies well below $M$ the theory given by (4.1) is indistinguishable from the renormalizable Abelian Higgs model. While some of these conditions can be naturally satisfied, for instance by polynomial TDF, the conditions related to the smallness of particle masses with respect to the Planck scale $M$ may require a fine-tuning (see Section 0). Hence, scale-invariant TDiff theories do not provide any explanation for the huge difference between the Planck mass $M$ and the mass scales of the SM. However, the presence of the extra field $\phi$ allows to introduce scale-invariant regularization schemes, under which the mass of the Higgs boson is not affected by the cut-off scale of the theory [12].

\section{Coupling to fermionic matter}

Finally, let us study the inclusion of fermions to scale-invariant TDiff theories ${ }^{24}$. A generic scale-invariant spinor Lagrangian compatible with TDiff can be written as ${ }^{25}$

$$
\mathcal{L}_{\psi}=-b \mathcal{G}_{\psi}\left(b^{2}\right) \bar{\psi} b^{\mu a} \gamma_{a}\left(\partial_{\mu}+\frac{1}{8}\left[\gamma_{c}, \gamma_{d}\right] \omega_{\mu}{ }^{c d}\right) \psi-b \phi v_{\psi}\left(b^{2}\right) \bar{\psi} \psi
$$

where $b^{\mu a}$ represents the inverse vierbein related to the metric through $g_{\mu \nu}=\eta_{a c} b_{\mu}{ }^{a} b_{\nu}{ }^{c}$, $\omega_{\mu}{ }^{c d}$ is its spin connection (see e.g. 445) and $b=\operatorname{det}\left[b_{\mu}{ }^{a}\right]=\sqrt{-g}$. Note that the fermionic fields have scaling dimension $d_{\psi}=3 / 2$. This is the most general Lagrangian if one requires polynomiality in the fields $\phi$ and $\psi$.

We should mention here that, as soon as a theory includes several fields with non-trivial scaling dimensions, scale invariance alone does not forbid the presence of arbitrary functions of scale-invariant field combinations. In the present example, all terms in the Lagrangian can in principle contain arbitrary functions of the combination $\bar{\psi} \psi / \phi^{3}$. Terms with $\phi$ in the denominator would be well-defined in a perturbative theory around a symmetry breaking background $\phi_{0} \neq 0$, however, they would correspond to higher dimensional operators. Terms with $\bar{\psi} \psi$ in the denominator, on the other hand, are in general ill-defined. We will

\footnotetext{
${ }^{24}$ For the sake of illustration we only consider Dirac spinors. Still the conclusions are generic as they only depend on the dimensionality of the fields. In this context see also 43, 44] for the first order formalism of unimodular gravity.

${ }^{25}$ In this section we use the conventions of 45 .
} 
stick to the requirement of polynomiality, bearing in mind that this is a variable dependent criterium.

Introducing the Stückelberg field as described in Section 2.1, the Lagrangian (5.1) can be written as

$$
\mathcal{L}_{\psi}=-b \mathcal{G}_{\psi}(\sigma) \bar{\psi} b^{\mu a} \gamma_{a}\left(\partial_{\mu}+\frac{1}{8}\left[\gamma_{c}, \gamma_{d}\right] \omega_{\mu}{ }^{c d}\right) \psi-b \phi v_{\psi}(\sigma) \bar{\psi} \psi
$$

In the vierbein formalism the field redefinition (3.11) corresponds to $\tilde{b}_{\mu}{ }^{a}=\Omega b_{\mu}{ }^{a}$. Together with the redefinition of the spinor field

$$
\tilde{\psi}=\Omega^{-3 / 2} \psi
$$

it yields the Lagrangian in the E-frame (see e.g. 45)

$$
\mathcal{L}_{\psi}=-\tilde{b} \mathcal{G}_{\psi}(\sigma) \overline{\tilde{\psi}} \tilde{b}^{\mu i} \gamma_{i}\left(\partial_{\mu}+\frac{1}{8}\left[\gamma_{j}, \gamma_{k}\right] \tilde{\omega}_{\mu}{ }^{j k}\right) \tilde{\psi}-\tilde{b} \frac{M v_{\psi}(\sigma)}{\sqrt{f(\sigma)}} \overline{\tilde{\psi}} \tilde{\psi}
$$

We see that the scale invariance of the spinor Lagrangian in the J-frame also leads to the decoupling of fermions from the dilaton field $\phi$ in the E-frame.

The above Lagrangian contains the interactions between the fundamental fermions, the gravitational field and the scalar field. To study non-relativistic processes, it is convenient to formulate these interactions in terms of particles interacting through certain potentials. For fields without strong interactions at low energies, this is done by a WKB approximation, and realizing that the corresponding particles propagate in geodesics of the metric to which they are coupled [46] (alternatively, one may use non-relativistic scattering amplitudes and the Born approximation to reconstruct the potential characterizing the interaction [47]). For other fields (such as quarks), the consequence of non-trivial couplings in lowenergy phenomenology (e.g. for the gravitational interaction of hadrons) is certainly more complicated 437, 48, 49. In the present case, it is relatively simple to write down the different possible terms that can appear for the point-particle Lagrangians. They will be of the form

$$
\mathcal{L}_{p p}=\int \mathrm{d} \tau \sqrt{\phi^{2} \mathcal{G}_{p p}(\sigma) g_{\mu \nu} \frac{\mathrm{d} x^{\mu}}{\mathrm{d} \tau} \frac{\mathrm{d} x^{\nu}}{\mathrm{d} \tau}}
$$

where $x^{\mu}(\tau)$ denotes the worldline of a point particle and $\mathcal{G}_{p p}(\sigma)$ is an arbitrary function to be deduced from (5.2). As happens for the fundamental fields, moving to the E-frame makes the field $\phi$ disappear ( $\phi$ is not coupled to matter fields), and we are back to a theory where particles move on geodesics of the effective metric $\mathcal{G}_{p p}(\sigma) \tilde{g}_{\mu \nu}$, reflecting the fact that the fundamental fields are coupled to the fields $\tilde{g}_{\mu \nu}$ and $\sigma$. The interaction mediated by $g_{\mu \nu}$ is long-ranged, while the range of the interaction due to $\sigma$ depends on its mass $m_{\varsigma}$ (cf. (4.10)).

\section{Application to the Standard Model}

The basics established in the preceding sections can be used to construct a scale-invariant version of the Standard Model of particle physics coupled to gravity. Let us describe how 
this should be done. The scalar-tensor sector of the theory is given by the Lagrangian (3.7) where $\phi$ is replaced by the complex Higgs-doublet $H$. All fermions and bosons of the SM are then added and coupled to gravity in the way described in Sections 1 and 5 , again with $H$ replacing $\phi$. The generalization to the group structure of the SM is straightforward. All TDF have to be chosen such that they fulfill a series of conditions of the type of (3.23), (4.11), (4.12), (4.15), 4.16), (4.19) and (4.22). In this way, one obtains a model whose particle phenomenology at energies well below the Planck mass $M$ is indistinguishable from that of the SM. In particular, the massless dilaton practically decouples from all the fields of the SM, except for the Higgs field to which it couples only through very suppressed interactions.

\section{Particular choices of the theory-defining functions}

In the previous sections we have derived a number of conditions to be satisfied by the theory-defining functions. These conditions are summarized in Table 1. Similar conditions should be imposed for the fermionic sector, but for the sake of simplicity we will restrict our considerations to the scalar and gauge sector (Lagrangian 4.1)).

\begin{tabular}{|l|l|c|}
\hline & \multicolumn{1}{|c|}{ Physical Meaning } & Formal Conditions \\
\hline C1 & Existence of a constant flat solution & $v\left(\sigma_{0}\right)=v^{\prime}\left(\sigma_{0}\right)=0$ \\
\hline C2 & Induced gravitational coupling & $f\left(\sigma_{0}\right) \neq 0$ \\
\hline C3 & $\begin{array}{l}\text { Positive definite kinetic terms } \\
\text { (absence of ghosts) }\end{array}$ & $\epsilon_{\varsigma}, \epsilon_{\varphi}, \epsilon_{A}=1$ \\
\hline C4 & $\begin{array}{l}\text { No negative masses } \\
\text { (absence of tachyons) }\end{array}$ & $m_{\varsigma}^{2}, m_{A}^{2} \geq 0$ \\
\hline C5 & Decoupling of gravitational interactions & $m_{\varsigma}, m_{A} \ll M$ \\
\hline C6 & No strong coupling & $\kappa_{\varsigma}, \kappa_{A} \lesssim \min \left(m_{\varsigma}, m_{A}\right)$ \\
\hline C7 & Suppression of higher-dimensional operators & $\lambda_{\phi \phi}, M_{V}, M_{i n t}, M_{A A}, M_{\varepsilon} \gtrsim M$ \\
\hline C8 & Equivalence with Abelian Higgs model & $\frac{\kappa_{A}}{\lambda_{A}} \simeq \frac{\kappa_{\varsigma}}{\lambda_{\varsigma}} \simeq 3 \frac{m_{\varsigma}^{2}}{\kappa_{\varsigma}} \simeq 2 \frac{m_{A}^{2}}{\kappa_{A}}$ \\
\hline
\end{tabular}

Table 1: Conditions to be imposed on the theory-defining functions (TDF)

The parameters in terms of which the conditions are formulated are defined through the TDF. They are summarized in Table 2 (remember that $\left.\tilde{e}^{2} \equiv \frac{e^{2}}{\mathcal{L}_{A A}^{(0)}}\right)$.

It is clear that it would be desirable to have an independent argument for choosing the arbitrary TDF (e.g. an additional symmetry) such that they automatically satisfy the conditions in Table 11. For the moment, we have unfortunately not found such a rationale. 


\begin{tabular}{|c|c|c|}
\hline i. & Signs of kinetic terms & $\begin{aligned} \epsilon_{\varsigma} & =\operatorname{sign}\left(\mathcal{K}_{\sigma \sigma}^{(0)}-\frac{\left(\mathcal{K}_{\sigma \phi}^{(0)}\right)^{2}}{\mathcal{K}_{\phi \phi}^{(0)}}-\frac{\left(\mathcal{K}_{\sigma A}^{(0)}\right)^{2}}{\mathcal{K}_{i n t}^{(0)}}\right) \\
\epsilon_{\varphi} & \equiv \operatorname{sign}\left(\mathcal{K}_{\phi \phi}^{(0)}\right) \\
\epsilon_{A} & \equiv \operatorname{sign}\left(\mathcal{K}_{A A}^{(0)}\right)\end{aligned}$ \\
\hline ii. & $\begin{array}{l}\text { Masses and } \\
\text { relevant couplings }\end{array}$ & $\begin{array}{lll}m_{\varsigma}^{2} \equiv \epsilon_{\varsigma} \tilde{V}^{(2)} M^{2}, & \kappa_{\varsigma} \equiv \tilde{V}^{(3)} M, & \lambda_{\varsigma} \equiv \tilde{V}^{(4)}, \\
m_{A}^{2} \equiv \epsilon_{A} \tilde{e}^{2} \mathcal{K}_{i n t}^{(0)} M^{2}, & \kappa_{A} \equiv \tilde{e}^{2} \tilde{\mathcal{K}}_{i n t}^{(1)} M, & \lambda_{A} \equiv \tilde{e}^{2} \tilde{\mathcal{K}}_{\text {int }}^{(2)},\end{array}$ \\
\hline iii. & Suppression scales & $\begin{array}{ll}M_{\phi \phi}\left(n_{h}, n_{\varsigma}\right) \sim M\left|\frac{\tilde{\mathcal{K}}_{\phi \phi}^{\left(n_{\varsigma}\right)}}{\tilde{\mathcal{K}}_{\phi \phi}^{(0)}}\right|^{\frac{-1}{n_{h}+n_{\varsigma}}}, & n_{h} \geq 0, n_{\varsigma}>0 \\
M_{V}\left(n_{h}, n_{\varsigma}\right) \sim M\left|\tilde{V}^{\left(n_{\varsigma}\right)}\right|^{\frac{-1}{n_{h}+n_{\varsigma}-4}}, & n_{h} \geq 0, n_{\varsigma}>4 \\
M_{i n t}\left(n_{h}, n_{\varsigma}\right) \sim M\left|\tilde{e}^{2} \tilde{\mathcal{K}}_{i n t}^{\left(n_{\varsigma}\right)}\right|_{-\frac{-1}{n_{h}+n_{\varsigma}}}, & n_{h} \geq 0, n_{\varsigma}>2 \\
M_{A A}\left(n_{h}, n_{\varsigma}\right) \sim M\left|\frac{\tilde{\mathcal{K}}_{A A}^{\left(n_{\varsigma}\right)}}{\tilde{\mathcal{K}}_{A A}^{(0)}}\right|^{\frac{-1}{n_{h}+n_{\varsigma}}}, & n_{h} \geq 0, n_{\varsigma}>0 \\
M_{\varepsilon}\left(n_{h}, n_{\varsigma}\right) \sim M\left|\frac{\tilde{\mathcal{K}}_{\varepsilon}^{\left(n_{\varsigma}\right)}}{\tilde{\mathcal{K}}_{A A}^{(0)}}\right|^{\frac{-1}{n_{h}+n_{\varsigma}}}, & n_{h} \geq 0, n_{\varsigma}>0\end{array}$ \\
\hline
\end{tabular}

Table 2: Relevant parameters appearing in Table 1

Nevertheless, we will give in this section three explicit ad hoc examples to show the existence of TDF satisfying the previous requirements.

\subsection{Polynomial TDF}

The first example we give is motivated by its simplicity. All theory-defining functions can be taken to be polynomials of the metric determinant. In analogy with the Higgs potential we choose

$$
v(-g)=\frac{\lambda}{4}\left(g_{0}^{2}-(-g)^{2}\right)^{2},
$$

which satisfies condition $\mathrm{C} 1$. The simplest choice for the remaining functions is given by

$$
\begin{aligned}
& f(-g)=\mathcal{G}_{g g}(-g)=\mathcal{G}_{A A}(-g)=1, \\
& \mathcal{G}_{g \phi}(-g)=\mathcal{G}_{n a}(-g)=\mathcal{G}_{\epsilon}(-g)=0, \\
& \mathcal{G}_{\phi \phi}(-g)=(-g)^{2} .
\end{aligned}
$$

For this choice of functions the parameters of the theory are summarized in Table $3\left(\sigma_{0}=\right.$ $\left.-g_{0}\right)$.

The conditions C1-C3 in Table 1 are immediately satisfied by this choice of TDF. The conditions $\mathrm{C} 4-\mathrm{C} 7$ are satisfied provided that $0<\sigma_{0} \ll 1$ and that $0<e^{2} \lesssim 1 / 2$ and $0<\lambda \lesssim 1 / 6$. Finally, the condition C8 always holds, independently of the parameter 


\begin{tabular}{|c|l|c|}
\hline i. & Signs of kinetic terms & $\epsilon_{\varsigma}=\epsilon_{\phi}=\epsilon_{A}=1$. \\
\hline ii. & Masses and relevant couplings & $\begin{array}{c}m_{\varsigma}^{2}=2 \lambda \sigma_{0}^{2} M^{2} \quad \kappa_{\varsigma}=6 \lambda \sigma_{0} M \quad \lambda_{\varsigma}=6 \lambda \\
m_{A}^{2}=e^{2} \sigma_{0}^{2} M^{2} \quad \kappa_{A}=2 e^{2} \sigma_{0} M \quad \lambda_{A}=2 e^{2}\end{array}$ \\
\hline iii. & Suppression scales & $M_{\phi \phi}\left(n_{h}, 1\right) \sim M\left(\frac{6+\sigma_{0}^{2}}{2 \sigma_{0}}\right)^{\frac{1}{1+n_{h}}}$, \\
& & $M_{\phi \phi}\left(n_{h}, 2\right) \sim M\left(\frac{6+\sigma_{0}^{2}}{2}\right)^{\frac{1}{2+n_{h}}}$. \\
\hline
\end{tabular}

Table 3: Parameters for TDF for Section 7.1.

values. The small value of $\sigma_{0}$ is responsible for the hierarchy between the Planck scale $M$ and the scales related to the scalar and vector sectors. It is also interesting to observe that the higher dimensional operators are suppressed below the Planck scale independently of the value of $\sigma_{0}$.

We conclude that the theory given by the Lagrangian (4.1) with TDF (7.1) and (7.2) is almost equivalent to the renormalizable Abelian Higgs model at energies well below the Planck scale $M$. The only difference is the term coming from the dilaton,

$$
\mathcal{L}_{d}=-\frac{1}{2} \sqrt{-\tilde{g}}\left(\left(\tilde{\sigma}+\sigma_{0}\right)^{2}+6\right)(\partial \tilde{\phi})^{2}
$$

The (non-renormalizable) interactions appearing in this term certainly produce differences between the two theories, but these effects are suppressed both by the Planck scale and by the derivative coupling of the dilaton. They may be relevant in the context of cosmology, discussed in the next section.

Finally, we would like to note that by changing variables one can easily find other sets of polynomial functions which describe a theory equivalent to the one given by (7.1) and (7.2) (and thus also to the Abelian Higgs model) and which also satisfy all conditions C1-C8. For example, one can redefine the metric and the scalar field $\phi$ through $^{26}$

$$
\begin{aligned}
g_{\mu \nu} & \mapsto(-g)^{2 \alpha} g_{\mu \nu}, \\
\phi & \mapsto(-g)^{\beta} \phi,
\end{aligned}
$$

where $\alpha$ and $\beta$ are some arbitrary numbers. In terms of the new variables the Lagrangian

\footnotetext{
${ }^{26}$ A slightly more general family equivalent to the Abelian Higgs models in the previous sense is easily found by allowing a generic function of $\tilde{\sigma}$ in $(7.3)$.
} 
(4.1) keeps its structure. The TDF equivalent to (7.1) and (7.2) are

$$
\begin{aligned}
& v(-g)=\frac{\lambda}{4}\left(g_{0}^{2+16 \alpha}-(-g)^{2+16 \alpha}\right)^{2}(-g)^{4(\alpha+\beta)} \\
& f(-g)=(-g)^{2(\alpha+\beta)} \\
& \mathcal{G}_{g g}(-g)=\left((1+8 \alpha)^{2}+\beta^{2}\right)(-g)^{18 \alpha+2 \beta}-\left(6 \alpha^{2}+12 \alpha \beta\right)(-g)^{2(\alpha+\beta)-2} \\
& \mathcal{G}_{\phi \phi}(-g)=(-g)^{18 \alpha+2 \beta+2} \\
& \mathcal{G}_{g \phi}(-g)=6 \alpha(-g)^{2(\alpha+\beta)-1}+\beta(-g)^{18 \alpha+2 \beta+1} \\
& \mathcal{G}_{A A}(-g)=1 \\
& \mathcal{G}_{n a}(-g)=\mathcal{G}_{\epsilon}(-g)=0
\end{aligned}
$$

It is straightforward to check explicitly that for $0<\left(-g_{0}\right)^{1+8 \alpha} \ll 1,0<e^{2} \lesssim 1 / 2$ and $0<\lambda \lesssim 1 / 6$ this set of polynomials also satisfies the conditions C1-C8. The two-parameter family of sets of functions (7.6) describes one and the same theory for different variable choices. For $\alpha=\beta=0$ the functions take the simple forms (7.1) and (7.2).

\subsection{TDF leading to Abelian Higgs model plus a decoupled dilaton}

In this example we show that one can choose TDF such that the particle physics part of the theory is exactly the Abelian Higgs model and the dilaton only couples to the gravitational field. To this end, we turn our attention to the Lagrangian in the form (4.3) and notice that if the TDF are such that

$$
\begin{aligned}
V(\sigma) & =\frac{\lambda}{4}\left(\sigma^{2}-\sigma_{0}^{2}\right)^{2} \\
f(\sigma) & =\sigma^{-1 / 4} \\
\mathcal{K}_{\sigma \sigma}(\sigma) & =\mathcal{K}_{\phi \phi}(\sigma)=\mathcal{K}_{A A}(\sigma)=1 \\
\mathcal{K}_{\sigma \phi}(\sigma) & =\mathcal{K}_{\sigma A}(\sigma)=\mathcal{K}_{\epsilon}(\sigma)=0 \\
\mathcal{K}_{i n t}(\sigma) & =\sigma^{2}
\end{aligned}
$$

that Lagrangian reads

$$
\begin{aligned}
\frac{\mathcal{L}_{e}}{\sqrt{-\tilde{g}}}= & -\frac{1}{2} M^{2} \tilde{R}-\frac{1}{2} M^{2}(\partial \sigma)^{2}-\frac{1}{2} M^{2}(\partial \ln (\phi / M))^{2}-\frac{1}{2} e^{2} M^{2} \sigma^{2} A^{2}-\frac{1}{4} F^{2} \\
& -M^{4} \frac{\lambda}{4}\left(\sigma^{2}-\sigma_{0}^{2}\right)^{2}-\frac{M^{4} \Lambda_{0}}{\phi^{4}} .
\end{aligned}
$$

For this particular case, the transformations (4.5) reduce to $\tilde{\sigma}=\sigma, \tilde{\phi}=M \ln \frac{\phi}{M}$ and $\tilde{A}_{\mu}=A_{\mu}$ and (4.6) becomes

$$
\begin{aligned}
\frac{\mathcal{L}_{e}}{\sqrt{-\tilde{g}}}= & -\frac{1}{2} M^{2} \tilde{R}-\frac{1}{2} M^{2}(\partial \tilde{\sigma})^{2}-\frac{1}{2}(\partial \tilde{\phi})^{2}-\frac{1}{2} e^{2} M^{2} \tilde{\sigma}^{2} \tilde{A}^{2}-\frac{1}{4} \tilde{F}^{2} \\
& -M^{4} \frac{\lambda}{4}\left(\tilde{\sigma}^{2}-\tilde{\sigma}_{0}^{2}\right)^{2}-\Lambda_{0} \exp \left(-\frac{4 \tilde{\phi}}{M^{4}}\right) .
\end{aligned}
$$


This is the Lagrangian of the Abelian Higgs model, $\tilde{\sigma}$ being the Higgs field in the unitary gauge, plus a dilaton field $\tilde{\phi}$ with an exponential potential proportional to $\Lambda_{0}$ and coupling only to gravity.

Making use of the relations (4.4) it is straightforward to find a set of TDF that satisfy the requirements $(7.7):{ }^{27}$

$$
\begin{aligned}
v(-g) & =\frac{\lambda}{4}(-g)^{-1 / 2}\left(g_{0}^{2}-(-g)^{2}\right)^{2} \\
f(-g) & =(-g)^{-1 / 4} \\
\mathcal{G}_{g g}(-g) & =(-g)^{-1 / 4}-\frac{3}{32}(-g)^{-9 / 4} \\
\mathcal{G}_{\phi \phi}(-g) & =(-g)^{7 / 4} \\
\mathcal{G}_{g \phi}(-g) & =\frac{3}{4}(-g)^{-5 / 4} \\
\mathcal{G}_{n a}(-g) & =-5(-g)^{-1 / 4}-(-g)^{7 / 4} \\
\mathcal{G}_{A A}(-g) & =1 \\
\mathcal{G}_{\epsilon}(-g) & =0
\end{aligned}
$$

By construction, this set of TDF satisfies all conditions C1-C8, as soon as $0<-g_{0} \ll 1$, $0<e^{2} \lesssim 1 / 2$ and $0<\lambda \lesssim 1 / 6$. Moreover, as the theory corresponds to the Abelian Higgs model, its particle physics part contains no higher dimensional operators and is renormalizable.

The choice of TDF given by (7.10) might seem somewhat peculiar. However, one should remember that the explicit expressions of the TDF depend on the variables in which one chooses to express the Lagrangian. In particular, if one chooses variables such that $d_{g}=2$ and $d_{\phi}=0$ (c.f. Section 3), the arbitrary functions only depend on $\phi$. In terms of those variables, the Abelian Higgs model plus decoupled dilaton corresponds to choosing the arbitrary functions to be polynomials in $\phi$.

\subsection{TDF reproducing scale-invariant unimodular gravity}

In an earlier work [5] two of us (M. S. and D. Z.) presented a model which combines scale invariance and unimodular gravity. There, a new singlet scalar field was introduced in order to make both the gravitational and the matter part of the action scale-invariant. Unlike in the present proposal, that scalar field was introduced ad hoc and was not related to the restriction of the gauge group from Diff to TDiff. Due to the shape of the potential, scale invariance was spontaneously broken. In the same model, standard GR was replaced by unimodular gravity with the aim of introducing a cosmological constant without explicitly breaking scale invariance. As already mentioned in Sections 1 and 2, the unimodular theory can be considered as a particular TDiff model with the constraint $g=-1$. Therefore, the model of 5 can certainly be written as a scale-invariant TDiff theory. To find the corresponding TDF, we will consider the simpler example where the full SM considered in

\footnotetext{
${ }^{27}$ Note that just like in the above example, this set of functions is only one representative of an infinite family of sets of functions that correspond to the same theory.
} 
(5) is replaced by the Abelian Higgs model, analyzed in the present paper. After choosing the TDF as ${ }^{28}$

$$
\begin{aligned}
& v(-g)=\frac{\lambda}{4}\left(2-\zeta^{2}(-g)^{-2}\right)^{2} \\
& f(-g)=\xi_{\chi}(-g)^{-2}+2 \xi_{h} \\
& \mathcal{G}_{g g}(-g)=\frac{49-90 \xi_{\chi}}{64}(-g)^{-4}+\frac{1+6 \xi_{h}}{32}(-g)^{-2}, \\
& \mathcal{G}_{\phi \phi}(-g)=2 \\
& \mathcal{G}_{g \phi}(-g)=-\frac{7-6 \xi_{\chi}}{8}(-g)^{-3}+\frac{1+6 \xi_{h}}{4}(-g)^{-1}, \\
& \mathcal{G}_{n a}(-g)=(-g)^{-2} \\
& \mathcal{G}_{A A}(-g)=1 \\
& \mathcal{G}_{\epsilon}(-g)=0
\end{aligned}
$$

the Lagrangian (4.1) can be brought to the form

$$
\begin{gathered}
\mathcal{L}_{S Z}=-\frac{1}{2}\left(\xi_{\chi} \chi^{2}+2 \xi_{h} \Phi \Phi^{*}\right) \hat{R}-\frac{1}{2} \hat{g}^{\mu \nu} \partial_{\mu} \chi \partial_{\nu} \chi-\hat{g}^{\mu \nu} D_{\mu} \Phi\left(D_{\nu} \Phi\right)^{*} \\
-\frac{1}{4} \hat{g}^{\mu \nu} \hat{g}^{\rho \sigma} F_{\mu \rho} F_{\nu \sigma}-\frac{\lambda}{4}\left(2 \Phi \Phi^{*}-\zeta^{2} \chi^{2}\right)^{2},
\end{gathered}
$$

where we have defined the unimodular metric $\hat{g}_{\mu \nu}=(-g)^{-1 / 4} g_{\mu \nu}$ and the scalar fields $\Phi=\phi(-g)^{1 / 8}$ and $\chi=|\phi|(-g)^{-7 / 8} . \quad \hat{R}$ is the Ricci scalar associated to the unimodular metric $\hat{g}_{\mu \nu}$. Note that for the variable change $\chi=|\phi|(-g)^{-7 / 8}$ to be well defined, $\chi$ is only allowed to take positive values. However, the theory being symmetric under $\chi \mapsto-\chi$, one can equally allow for negative values of $\chi$. In that part of phase space the matching of the variables is $\chi=-|\phi|(-g)^{-7 / 8}$. We see that $(7.12)$ is exactly the Lagrangian of the model proposed in $\|5\|$ reduced to the Abelian Higgs model.

As for the choice of TDF discussed in the previous subsection, the choice of functions (7.11) is rather peculiar and in particular, the presence of the non-analytic term $\mathcal{G}_{n a} \neq 0$ in (4.1) is essential to find the Lagrangian $\mathcal{L}_{S Z}$. Again, there exists a set of variables, $\hat{g}_{\mu \nu}$, $\phi$ and $\chi$, in terms of which the expression of the Lagrangian becomes particularly simple.

The complex scalar field $\Phi$ in (7.12) plays the role of the Higgs field, non-minimally coupled to gravity. If one includes fermions, then this is the field that couples to fermions through Yukawa couplings. The real scalar field $\chi$ is a kind of dilaton. The flat direction in the potential guarantees that the theory possesses an infinite family of ground states which spontaneously break the dilatational symmetry. In [5] it was shown that the Lagrangian (7.12) (if one adds all SM matter and gauge fields) represents a viable model for SM phenomenology which besides enforces interesting cosmological phenomena if the parameters are positive and such that $\zeta \lll 1, \xi_{\chi} \sim \mathcal{O}\left(10^{-3}\right), \xi_{h} \sim \mathcal{O}\left(10^{5}\right)$ and $\lambda \lesssim 1$. The smallness of $\zeta$ is responsible for the hierarchy between the Planck scale and the electroweak scale. The values of $\xi_{\chi}$ and $\xi_{h}$ are fixed by cosmological considerations (cf. 河) .

\footnotetext{
${ }^{28}$ Like in the above examples, this set of functions is only one representative of an infinite family of sets of functions that correspond to the same theory.
} 


\begin{tabular}{|c|l|c|}
\hline i. & Signs of kinetic terms & $\epsilon_{\varsigma}=\epsilon_{\phi}=\epsilon_{A}=1$. \\
\hline & & $m_{\varsigma}^{2}=2 \lambda \frac{\zeta^{2}}{\xi_{\chi}} M^{2}\left(1+\mathcal{O}\left(\zeta^{2}\right)\right)$, \\
& & $\kappa_{\varsigma}=6 \lambda \sqrt{\frac{\zeta^{2}}{\xi_{\chi}}} M\left(1+\mathcal{O}\left(\zeta^{2}\right)\right)$, \\
ii. & Masses and relevant couplings & $\lambda_{\varsigma}=6 \lambda\left(1+\mathcal{O}\left(\zeta^{2}\right)\right)$, \\
& & $m_{A}^{2}=e^{2} \frac{\zeta^{2}}{\xi_{\chi}} M^{2}\left(1+\mathcal{O}\left(\zeta^{2}\right)\right)$, \\
& & $\kappa_{A}=2 e^{2} \sqrt{\frac{\zeta^{2}}{\xi_{\chi}}} M\left(1+\mathcal{O}\left(\zeta^{2}\right)\right)$, \\
& & $\lambda_{A}=2 e^{2}\left(1+\mathcal{O}\left(\zeta^{2}\right)\right)$. \\
\hline iii. & Suppression scales & $M_{\phi \phi}, M_{V}, M_{i n t}, M_{A A} \sim \frac{M}{\xi_{h}}<M$. \\
\hline
\end{tabular}

Table 4: Parameters the scale-invariant unimodular gravity (Section 7.2).

Let us now check, whether the model given by (7.11) satisfies the conditions C1-C8 appearing in Table 1. To this end, we consider the expansion of the different functions expanded around a constant solution $g_{0}=\sigma_{0}=\frac{\sqrt{2}}{\zeta}$. The different parameters are summarized in Table 1 . For the phenomenologically interesting parameters, conditions C1-C6 and $\mathrm{C} 8$ hold. We are left with the question about higher dimensional operators. In the present example, all terms of (4.6), except the one proportional to $\tilde{\mathcal{K}}_{\epsilon}(\tilde{\sigma})=0$ and the one proportional to $\Lambda_{0}=0$, give rise to an infinite number of higher dimensional operators. Depending on the values of the parameters, their suppression scales can be smaller than the Planck scale $M$. For the phenomenologically interesting parameters, the lowest suppression scales are of the order $\frac{M}{\xi_{h}}$. Although significantly smaller than the Planck scale, this scale is still much higher then the scales relevant to particle physics and can be consider as the cut-off scale of the theory ${ }^{29}$. Although condition $\mathrm{C} 7$ is not exactly satisfied, the higher dimensional operators are still negligible at particle physics scales and the rest of conditions are fulfilled to high accuracy. We conclude that at energies well below $\frac{M}{\xi_{h}}$ the theory given by the Lagrangian (4.1) with defining functions (7.11) (respectively the equivalent theory (7.12) ) is also indistinguishable from the renormalizable Abelian Higgs model.

\section{The case $\Lambda_{0} \neq 0$, cosmology and dilaton interactions}

So far we have mainly considered static backgrounds for which (3.23) is satisfied and $\Lambda_{0}=0$. The first condition is about the TDF. It is equivalent to the absence of a cosmological constant (cf. Section 3.1). The second condition is about the choice of the initial state. It is related to the TDiff invariance and has nothing to do with the TDF. Some motivations for the first condition in (3.23), valid when gravity is dropped of (i.e. neglecting the scalar curvature term $R$ in the Jordan frame action) were given in [12]. Namely, if $v\left(-g_{0}\right)>0$,

\footnotetext{
${ }^{29}$ Note that this lowering of the physical cut-off scale below the Planck scale happens is generic in models where the Higgs field is non-minimally coupled to gravity. For a recent discussion on this issue see [10].
} 
the ground state of the system is scale-invariant, meaning that the theory does not have any particle excitations or that the theory is free. If $v\left(-g_{0}\right)<0$, the theory does not have a ground state at all. In other words, the only sensible case is $v\left(-g_{0}\right)=0$ corresponding to a flat direction in the scalar potential and leading to spontaneous breakdown of scale invariance. As we have seen in Section 3, if gravity is included, the cases where $v\left(-g_{0}\right) \neq 0$ do not have known pathologies and simply correspond to dS or AdS spaces, characterized by a non-zero cosmological constant. So, scale-invariant TDiff theories do not give a solution to the cosmological constant problem. Still, they provide another perspective towards its solution, transferring the problem to the requirement of some specific property (eq. (3.23)) of one of the TDF.

We will start this section by considering that $(3.23)$ is satisfied, but $\Lambda_{0} \neq 0$. Let us discuss qualitatively the cosmological solutions in our theories and see how they affect local particle physics. To this end we consider the Lagrangian (3.17) to which we add a matter part

$$
\begin{aligned}
\frac{\mathcal{L}_{e}}{\sqrt{-\tilde{g}}}= & -\frac{1}{2} M^{2} \tilde{R}-\frac{1}{2} \epsilon_{\sigma} M^{2}(\partial \tilde{\sigma})^{2}-\frac{1}{2} \tilde{\mathcal{K}}_{\phi \phi}(\tilde{\sigma})(\partial \tilde{\phi})^{2} \\
& -M^{4} \tilde{V}(\tilde{\sigma})-\Lambda_{0} \tilde{\mathcal{K}}_{\Lambda_{0}}(\tilde{\sigma}) \exp \left(-\frac{4 \tilde{\phi}}{M}\right)+\mathcal{L}_{m}
\end{aligned}
$$

where $\mathcal{L}_{m}$ contains all bosonic and fermionic degrees of freedom of the SM coupled to the scalar fields and gravity in the way described in Sections 1 and 5 . Notice that the dependence of the potential on $\tilde{\phi}$ is uniquely determined by the way scale invariance is broken in TDiff theories. Similar potentials have been considered in the past in the context of scalar tensor theory, cf. [45]. Consider now the homogeneous fields $\tilde{\sigma}=\tilde{\sigma}(t)$ and $\tilde{\phi}=\tilde{\phi}(t)$ living in a flat Friedmann-Lemaître-Robertson-Walker (FLRW) space-time with metric

$$
\mathrm{d} \tilde{s}^{2}=-\mathrm{d} t^{2}+\tilde{a}(t)^{2} \mathrm{~d} \vec{x}^{2}
$$

where $\tilde{a}(t)$ is the scale factor. The dynamics of the homogeneous scalar fields is mainly determined by the potential

$$
\tilde{V}_{\Lambda_{0}}(\tilde{\sigma}, \tilde{\phi})=M^{4} \tilde{V}(\tilde{\sigma})+\Lambda_{0} \tilde{\mathcal{K}}_{\Lambda_{0}}(\tilde{\sigma}) \exp \left(-\frac{4 \tilde{\phi}}{M}\right) .
$$

As long as the kinetic term of the scalar fields is positive-definite, the scalar fields tend to roll down the potential, with some friction caused by the expansion of space-time. In the $\tilde{\sigma}$-direction the potential has a minimum at $\tilde{\sigma}=0$ due to the conditions $(4.7)^{30}$. In the $\tilde{\phi}$-direction, the potential is governed by the exponential factor. If $\Lambda_{0} \tilde{\mathcal{K}}_{\Lambda_{0}}(\tilde{\sigma})>0$, the potential is of the run-away type, i.e. it gets minimal for $\tilde{\phi} \rightarrow \infty$. In the opposite pathological - case the potential for $\tilde{\phi}$ is not bounded from below. Hence, a typical evolution of the scalar condensates $\tilde{\sigma}$ and $\tilde{\phi}$ will be the following: The first term of the potential $\tilde{V}_{\Lambda_{0}}$ drives the trajectories towards the "valley" $\tilde{\sigma}=0$. Due to the Hubble friction the field

\footnotetext{
${ }^{30}$ Note that to get this minimum it is enough that condition (3.21) holds.
} 
undergoes damped oscillations around the valley before asymptotically approaching $\tilde{\sigma}=0$. The second term in $\tilde{V}_{\Lambda_{0}}$ drives the trajectory towards $\tilde{\phi} \rightarrow \infty$. After $\tilde{\sigma}$ has settled down in the valley, this leads to a roll-down along the valley ${ }^{31}$.

For appropriate choices of the TDF and initial conditions, the roll-down towards the valley $\tilde{\sigma}=0$ can give a mechanism for inflation. During the subsequence roll-down along the valley, the scalar fields can play the role of a dynamical dark-energy component (quintessence). This is a generic scenario for scale-invariant TDiff theories. A concrete realization has been proposed in $[5]$ (see also 50 ).

Since the evolution drives $\tilde{\sigma} \rightarrow 0$ it seems reasonable to assume that in the present universe $\tilde{\sigma} \simeq 0$. If this is fulfilled, then all masses and couplings of the SM-particles are like in the case $\Lambda_{0}=0$ described in the above sections. The only effects of the cosmological background on particle physics would then come through $\tilde{\phi}(t)$. One can put simple and still very strong bounds on the influence of $\tilde{\phi}(t)$ by requiring that its energy density does not give a too big contribution to the energy density of the universe. In other words, both the kinetic and the potential energy of the condensate $\tilde{\phi}(t)$ have to be smaller than today's critical energy density $\rho_{c r}^{0}=3 M^{2} H_{0}^{2} \simeq 10^{-120} M^{4}$, i.e.

$$
\begin{aligned}
& \frac{1}{2} \tilde{\mathcal{K}}_{\phi \phi}\left(\tilde{\sigma}_{0}=0\right)\left(\partial_{0} \tilde{\phi}\right)^{2}<\rho_{c r}^{0}, \\
& \Lambda_{0} \tilde{V}\left(\tilde{\sigma}_{0}=0\right) \exp \left(-\frac{4 \tilde{\phi}}{M}\right)<\rho_{c r}^{0} .
\end{aligned}
$$

These constraints, together with the conditions on the derivatives of $\tilde{\mathcal{K}}_{\phi \phi}(\tilde{\sigma}),(4.19)$ and similar conditions on the derivatives of $\tilde{V}_{\Lambda_{0}}(\tilde{\sigma})$ guarantee that all interactions induced by $\partial_{0} \tilde{\phi} \neq 0$ are highly suppressed and can be neglected in the description of local particle interactions.

Finally, we would like to briefly comment on the situation where $v\left(\sigma_{0}\right) \neq 0$. As we already stated, this case is equivalent to the presence of a cosmological constant (cf. (3.22)). In this situation, phenomenological bounds imply that this term must be very small, and will not affect the conclusions on local physics of the previous sections. It will, however, be important for late time cosmology, as it represents a contribution to dark energy on top of those coming from the dynamics of the scalar fields presented previously in this section. In fact, asymptotically, this constant term becomes dominant over the other contributions, as they are diluted during the expansion of the universe.

\section{Conclusions}

In this paper we have shown that scale-invariant TDiff theories constitute a viable alternative to standard General Relativity (GR). The group of space-time symmetries of these theories is not the full group of diffeomorphisms, but rather its subgroup defined by 4volume preserving transformations. Hence, TDiff theories depend on a number of a priori

\footnotetext{
${ }^{31}$ We neglect here effects of potential-terms involving couplings of $\tilde{\sigma}$ to the SM-fields. Also, we assumed that the function $\tilde{\mathcal{K}}_{\Lambda_{0}}$ defined in (3.18) does not play a significant role in the cosmological evolution.
} 
arbitrary functions of the metric determinant, the theory-defining functions (TDF). As a consequence, TDiff theories generically have more physical degrees of freedom in the gravitational sector: in addition to the massless graviton they contain a propagating scalar degree of freedom that may or may not be massive.

In order to study the phenomenology of TDiff theories, we first formulated them in terms of equivalent Diff invariant theories by means of a Stückelberg field. An advantage of the Stückelberg formalism is that it makes the new scalar field appear explicitly in the Lagrangian. A very interesting feature of TDiff theories is the appearance of an arbitrary mass scale $\Lambda_{0}$. In the TDiff formulation of the theory this scale appears as an integration constant in the equations of motion, while in the equivalent Diff invariant formulation it appears as a new coupling constant in the Lagrangian. The appearance of $\Lambda_{0}$ is exactly analog to the appearance of an arbitrary cosmological constant in unimodular gravity. Notice, however, that in the present context $\Lambda_{0}$ does not play the role of a cosmological constant.

Next, we focused on the scale-invariant case, i.e. we considered actions that are invariant under global dilatations. We were interested in the situation where scale invariance is spontaneously broken, such that all scales of the theory are induced by the expectation value of a scalar field. We found that if the theory contains only one scalar degree of freedom, this degree of freedom is necessarily the Goldstone boson of the spontaneously-broken scale invariance. Therefore, as our objective was to construct a theory in which a scalar field plays the role of the SM Higgs field, we were lead to the introduction of an additional scalar field. The scale-invariant TDiff theories including an additional real scalar field were studied in detail. After extending the scale-invariant TDiff theories to gauge theories and including fermionic fields, we discussed how the framework can be generalized to include all degrees of freedom of the Standard Model.

For $\Lambda_{0}=0$, the spectrum of scalar excitations around a symmetry-breaking background consists of the a massless scalar dilaton plus a potentially massive scalar degree of freedom. The dilaton decouples from all SM degrees of freedom except for the Higgs field, to which it couples derivatively. A non-zero $\Lambda_{0}$ leads to a very particular potential term breaking the scale symmetry explicitly. This term can yield an additional interaction between the dilaton and the Higgs field which is, however, negligible for particle physics phenomenology. Interestingly, the $\Lambda_{0}$-term can depend on the dilaton only through the exponential function $\exp (-4 \tilde{\phi} / M)$. As a consequence, the dilaton can give rise to dynamical dark energy.

For the theory restricted to the gravitational, vector and scalar sectors, we derived the conditions on the TDF leading to a renormalizable low-energy theory in the particle physics sector. Moreover, we gave three explicit examples of TDF satisfying these conditions. One of the examples corresponds to the model of $[5]$.

Next, we commented on the generic behavior of cosmological solutions. In particular, we found that the conditions yielding a theory close to the SM, entail an interesting cosmological phenomenology. Namely, the corresponding solutions can describe a phase of inflation in the very early universe, whereas the existence of a small $\Lambda_{0}$ produces a runaway potential for the dilaton, which can hence play the role of a dynamical dark energy component. 
Finally, generic TDF also imply the presence of a pure cosmological constant term. Keeping this contribution small represents a fine-tuning similar to the fine-tuning required in GR to get a small cosmological constant. Indeed, it is interesting to compare this situation with the naturalness problem of the Higgs mass. Scale invariance is a key ingredient for a solution of the problem of stability of the Higgs mass against radiative corrections. This invariance keeps the corrections small (at least if dimensional regularization is used [12]). At the same time, the smallness of the Higgs mass in comparison with the Planck scale is not explained and must be imposed "by hand", as in SM with a cut-off given by the scale of strong coupling of GR. The same statement is true for the cosmological constant. In spite of the fact that scale invariance forbids any mass parameters in a theory, a cosmological constant is generally present, and is related to the self-interaction of a scalar field (Higgs) in the J-frame. Only tuning this term to zero makes for the absence of a cosmological constant.

\section{Acknowledgements}

This work was supported by the Swiss National Science Foundation and by Tomalla Foundation. We thank Jaume Garriga and Sergey Sibiryakov for helpful discussions.

\section{References}

[1] P. Minkowski, Phys. Lett. B 71 (1977) 419.

[2] A. Zee, Phys. Rev. Lett. 42 (1979) 417.

[3] J. L. Cervantes-Cota and H. Dehnen, Nucl. Phys. B 442 (1995) 391 [arXiv:astro-ph/9505069].

[4] J. J. van der Bij, Acta Phys. Polon. B 25 (1994) 827.

[5] M. Shaposhnikov and D. Zenhausern, Phys. Lett. B 671 (2009) 187 [arXiv:0809.3395 [hep-th]].

[6] Y. Fujii, Phys. Rev. D 26 (1982) 2580.

[7] C. Wetterich, Nucl. Phys. B 302 (1988) 645.

[8] C. Wetterich, Nucl. Phys. B 302 (1988) 668.

[9] F. L. Bezrukov, A. Magnin and M. Shaposhnikov, Phys. Lett. B 675 (2009) 88 [arXiv:0812.4950 [hep-ph]].

[10] F. Bezrukov, A. Magnin, M. Shaposhnikov, S. Sibiryakov, JHEP 1101, 016 (2011). [arXiv:1008.5157 [hep-ph]].

[11] F. Englert, C. Truffin and R. Gastmans, Nucl. Phys. B 117 (1976) 407.

[12] M. Shaposhnikov and D. Zenhausern, Phys. Lett. B 671 (2009) 162 [arXiv:0809.3406 [hep-th]].

[13] M. E. Shaposhnikov and I. I. Tkachev, Phys. Lett. B 675 (2009) 403 [arXiv:0811.1967 [hep-th]].

[14] M. E. Shaposhnikov and F. V. Tkachov, arXiv:0905.4857 [hep-th]. 
[15] P. Jain, S. Mitra and N. K. Singh, JCAP 0803 (2008) 011 [arXiv:0801.2041 [astro-ph]].

[16] J. J. van der Bij, H. van Dam and Y. J. Ng, Physica 116A (1982) 307.

[17] F. Wilczek, Phys. Rept. 104 (1984) 143.

[18] A. Zee, Proc. of 20th Annual Orbis Scientiae, Plenum, NY 1985, p. 211.

[19] W. Buchmuller and N. Dragon, Phys. Lett. B 207 (1988) 292.

[20] S. Weinberg, Rev. Mod. Phys. 61 (1989) 1.

[21] W. G. Unruh, Phys. Rev. D 40 (1989) 1048.

[22] E. Alvarez, JHEP 0503 (2005) 002.

[23] M. Henneaux and C. Teitelboim, Phys. Lett. B 222 (1989) 195.

[24] R. P. Feynman, F. B. Morinigo and W. G. Wagner, Eur. J. Phys. 24 (2003) 330.

[25] E. Alvarez, D. Blas, J. Garriga and E. Verdaguer, Nucl. Phys. B 756 (2006) 148 [arXiv:hep-th/0606019].

[26] C. M. Will, Living Rev. Rel. 9 (2005) 3 [arXiv:gr-qc/0510072].

[27] D. Blas, O. Pujolas and S. Sibiryakov, arXiv:1007.3503 [hep-th].

[28] V. A. Rubakov and P. G. Tinyakov, Phys. Usp. 51 (2008) 759 [arXiv:0802.4379 [hep-th]].

[29] Yu. F. Pirogov, arXiv:gr-qc/0609103.

[30] E. Alvarez, R. Vidal, Phys. Rev. D81, 084057 (2010). [arXiv:1001.4458 [hep-th]].

[31] E. Alvarez and A. F. Faedo, Phys. Rev. D 76 (2007) 064013 [arXiv:hep-th/0702184].

[32] J. J. Lopez-Villarejo, arXiv:1009.1023 [hep-th].

[33] E. I. Guendelman and A. B. Kaganovich, AIP Conf. Proc. 861 (2006) 875 [arXiv:hep-th/0603229].

[34] B. Fiol and J. Garriga, JCAP 1008 (2010) 015 [arXiv:0809.1371 [hep-th]].

[35] Y. J. Ng and H. van Dam, Phys. Rev. Lett. 65 (1990) 1972.

[36] J. D. Barrow and D. J. Shaw, Phys. Rev. Lett. 106 (2011) 101302 [arXiv:1007.3086 [gr-qc]].

[37] T. Damour and J. F. Donoghue, Phys. Rev. D 82 (2010) 084033 [arXiv:1007.2792 [gr-qc]].

[38] J. R. Ellis, N. C. Tsamis and M. B. Voloshin, Phys. Lett. B 194 (1987) 291.

[39] S. R. Coleman, J. Wess and B. Zumino, Phys. Rev. 177 (1969) 2239.

[40] J. M. Cornwall, D. N. Levin and G. Tiktopoulos, Phys. Rev. D 10 (1974) 1145 [Erratum-ibid. D 11 (1975) 972].

[41] M. N. Chernodub, L. Faddeev and A. J. Niemi, JHEP 0812 (2008) 014 [arXiv:0804.1544 [hep-th]].

[42] L. D. Faddeev, arXiv:0811.3311 [hep-th].

[43] D. Blas, Class. Quant. Grav. 25 (2008) 154009 [arXiv:0803.4497 [hep-th]].

[44] D. Blas, arXiv:0809.3744 [hep-th].

[45] Y. Fujii and K. Maeda, Cambridge, USA: Univ. Pr. (2003) $240 p$ 
[46] E. Alvarez, A. F. Faedo and J. J. Lopez-Villarejo, JHEP 0810 (2008) 023 [arXiv:0807.1293 [hep-th]].

[47] N. E. J. Bjerrum-Bohr, arXiv:hep-th/0410097.

[48] D. B. Kaplan and M. B. Wise, JHEP 0008 (2000) 037 [arXiv:hep-ph/0008116].

[49] L. Hui and A. Nicolis, Phys. Rev. Lett. 105 (2010) 231101 [arXiv:1009.2520 [hep-th]].

[50] J. Garcia-Bellido, J. Rubio, M. Shaposhnikov and D. Zenhausern, in preparation. 